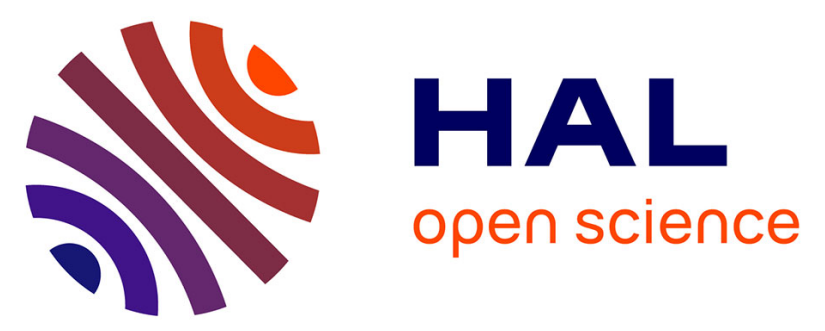

\title{
Experimental density data of three carbon dioxide and oxygen binary mixtures at temperatures from 276 to 416 $\mathrm{K}$ and at pressures up to $20 \mathrm{MPa}$
}

Snaide Ahamada, Alain Valtz, Salaheddine Chabab, Laura Blanco Martín, Christophe Coquelet

\section{To cite this version:}

Snaide Ahamada, Alain Valtz, Salaheddine Chabab, Laura Blanco Martín, Christophe Coquelet. Experimental density data of three carbon dioxide and oxygen binary mixtures at temperatures from 276 to $416 \mathrm{~K}$ and at pressures up to $20 \mathrm{MPa}$. Journal of Chemical and Engineering Data, 2020, 65 (11), pp.5313-5327. 10.1021/acs.jced.0c00484 . hal-03001731

\section{HAL Id: hal-03001731 \\ https://hal.science/hal-03001731}

Submitted on 12 Nov 2020

HAL is a multi-disciplinary open access archive for the deposit and dissemination of scientific research documents, whether they are published or not. The documents may come from teaching and research institutions in France or abroad, or from public or private research centers.
L'archive ouverte pluridisciplinaire HAL, est destinée au dépôt et à la diffusion de documents scientifiques de niveau recherche, publiés ou non, émanant des établissements d'enseignement et de recherche français ou étrangers, des laboratoires publics ou privés. 
Experimental density data of three carbon dioxide and oxygen binary mixtures at temperatures from 276 to $416 \mathrm{~K}$ and at pressures up to $20 \mathrm{MPa}$

Snaide Ahamada ${ }^{\mathrm{a}}$, Alain Valtz ${ }^{\mathrm{a}}$, Salaheddine Chabab $^{\mathrm{a}}$, Laura Blanco-Martín ${ }^{\mathrm{b}}$, Christophe Coquelet $^{\mathrm{a}}$

a MINES ParisTech PSL University, CTP-Centre of Thermodynamics of Processes, 35, Rue Saint Honoré, 77305 Fontainebleau, France

b MINES ParisTech PSL University, Department of Geosciences, 35, Rue Saint Honoré, 77305 Fontainebleau, France

* Corresponding Author: Christophe Coquelet (Christophe.coquelet@mines-paristech.fr) Tel: +33164694962 Fax: +33164694968 


\begin{abstract}
In the context of Power-to-Gas systems, including Power-to-Gas-Oxyfuel, possible storage of a mixture of $\mathrm{CO}_{2}$ and $\mathrm{O}_{2}$ requires density data information and evaluation of equations of state. Densities of three $\mathrm{CO}_{2}-\mathrm{O}_{2}$ binary system were measured using a vibrating tube densitometer (VTD), and the forced path mechanical calibration (FPMC) method in the gas, liquid and supercritical regions between 276 and $416 \mathrm{~K}$ and at pressures up to $20 \mathrm{MPa}$ (maximum expanded uncertainties $U(p)=0.0005 \mathrm{MPa}, U(T)=0.3 \mathrm{~K}$ and $U(\rho)=15 \mathrm{~kg} \cdot \mathrm{m}^{-3}$ ). The mole fractions of the prepared $\mathrm{CO}_{2} / \mathrm{O}_{2}$ mixtures are $0.726 / 0.274,0.517 / 0.483$ and 0.872/0.128. The Peng-Robinson cubic equation of state (PR EoS) and the EoS-CG, based on GERG-2008 (and implemented in Refprop v10.0), were considered for the analysis of the data. Comparisons were done with literature data. It appears that the data are overall better predicted by the EoS-CG than the PR EoS.
\end{abstract}

\title{
List of symbols
}

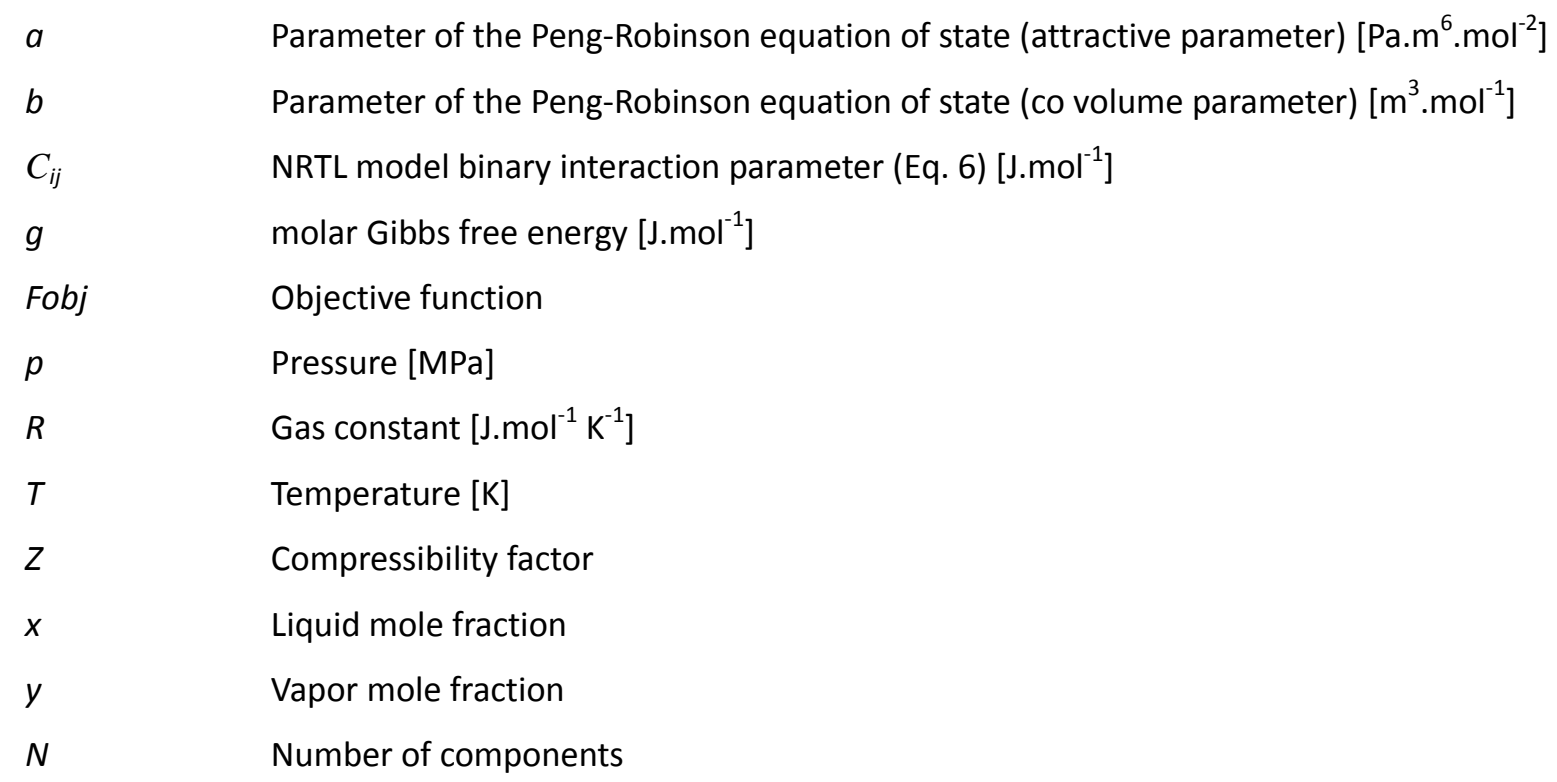

\section{Greek letters}




$\begin{array}{ll}\alpha_{i j} & \text { NRTL model parameter (Eq. 6) } \\ \omega & \text { Acentric factor } \\ \Delta & \text { Deviation }\end{array}$

\section{Superscript}

E

Excess property

\section{Subscripts}

$\begin{array}{ll}C & \text { Critical property } \\ \text { cal } & \text { Calculated property } \\ \exp & \text { Experimental property } \\ i, j & \text { Molecular species } \\ v & \text { Vapor phase } \\ I & \text { liquid phase }\end{array}$




\section{Introduction}

In the context of energy transition from fossil to low-carbon energy, the Power-to-Gas concept seems to be a very promising solution ${ }^{1}$. It consists of a transformation of $\mathrm{CO}_{2}$ with $\mathrm{H}_{2}$, produced by water electrolysis using renewable electricity, into methane, $\mathrm{CH}_{4}$ (methanation: Sabatier reaction). Methane can be used as a fuel or can be transformed into electricity (oxyfuel combustion for example ${ }^{2}$ ). Due to the intermittent nature of renewable sources of energy like solar or wind, it is important to develop solutions for massive energy storage. Massive energy storage is necessary to succeed in the energy transition and a solution consists of storage in salt caverns. The design of salt caverns requires thermodynamic properties such as gas solubility in brine and also volumetric properties of the stored products. In Power-to-Gas-Oxyfuel, storage of $\mathrm{CO}_{2}, \mathrm{O}_{2}$ and $\mathrm{CH}_{4}$ is required. In general, products are stored in single-phase conditions in salt caverns, but in the case of $\mathrm{CO}_{2}$, phase changes are possible as its critical pressure and temperature are in the range of typical storage conditions. In order to overcome the problem, one solution would be to mix $\mathrm{CO}_{2}$ and $\mathrm{O}_{2}$ in the same salt cavern ${ }^{3}$. As $\mathrm{O}_{2}$ is a cryogenic fluid, a mixture of $\mathrm{CO}_{2}$ with $\mathrm{O}_{2}$ will lead to a lower value of critical temperature. This way the mixture will stay in single-phase conditions.

It exists several sets of data in the open literature concerning the density of mixtures of $\mathrm{CO}_{2}$ and $\mathrm{O}_{2}$. Li et al. ${ }^{4}$ have published in 2019 a very complete review concerning the available thermo-physical properties of $\mathrm{CO}_{2}$ mixtures.in the context of $\mathrm{CO}_{2}$ capture and storage (CCS). They have mentioned in their paper all the available references concerning the VLE and density properties of the $\mathrm{CO}_{2}+\mathrm{O}_{2}$ binary system. We can also cite the works of Lozano-Martín et al. ${ }^{5}$, Commodore et al. ${ }^{6}$ and Mantovani et al. ${ }^{7}$. In these investigations, the composition in $\mathrm{O}_{2}$ is lower than 0.2.

In this work, the densities of three $\mathrm{CO}_{2}-\mathrm{O}_{2}$ mixtures were measured using Vibrating Tube Densitometer (VTD) in the gas, liquid and supercritical regions. A wide range of $\mathrm{O}_{2}$ molar fractions was investigated. The measurements were carried out at eight isotherms between 276 and $416 \mathrm{~K}$ at pressures up to $20 \mathrm{MPa}$. The measured densities were also employed to evaluate the capability of a cubic Equation of State (EoS) to predict the density of the binary mixtures. The cubic EoS is composed of the Peng-Robinson (PR-EoS) associated with a $g^{E}$ mixing rule. Additionally, a recent EoS based on GERG-2008, EOS-CG ${ }^{8}$, was evaluated using 
the measured density data and derived thermodynamic properties (compressibility factor). The experimental data obtained successfully compares to available density data in the literature.

\section{Experimental part}

\subsection{Materials}

$\mathrm{CO}_{2}$ and $\mathrm{O}_{2}$ were purchased from Air Liquide with a purity higher than 99.995 vol.\% and 99.999 vol.\% (Table 1). Table 2 presents the exact composition of the three mixtures. The mixtures were prepared in a gas reservoir considering the difference of total pressure. $\mathrm{CO}_{2}$ was first introduced into the gas reservoir under vacuum. Pressure was recorded $(P 1)$. Afterwards, $\mathrm{O}_{2}$ is introduced and pressure is recorded $(P 2)$. The temperature of the gas reservoir is selected in order to have a monophasic phase inside. Approximate composition is estimated using $x_{O 2}=(P 2-P 1) / P 2$.

For more accuracy, the compositions were determined by means of a Gas Chromatograph analysis (Varian, model CP 3800), using a thermal conductivity detector (TCD). WINILAB III software (Perichrom, France) is used for peaks integration and their analysis. The calibration of the GC detector is made by introducing known pure component volumes with appropriate syringes. The packed column used in the gas chromatograph is a PORAPAK R (80/100 mesh, $1.2 \mathrm{~m} \mathrm{X} \mathrm{1/8"} \mathrm{Silcosteel)} \mathrm{column.} \mathrm{A} \mathrm{calibration} \mathrm{curve} \mathrm{between} \mathrm{moles} \mathrm{number} \mathrm{introduced} \mathrm{and}$ GC peak surface is determined and considered to estimate the accuracies. The resulting relative accuracies concerning the mole numbers are $1.1 \%$ for $\mathrm{CO}_{2}$ and $1.2 \%$ for $\mathrm{O}_{2}$ for mixture 2 and $0.7 \%$ for $\mathrm{CO}_{2}$ and $1.6 \%$ for $\mathrm{O}_{2}$ for mixtures 1 and 3 . The uncertainty of molar fractions $\left(x_{1}\right)$ is determined by Eq. (1):

$u\left(x_{1}\right)=x_{1}\left(1-x_{1}\right) \sqrt{\left(\frac{u\left(n_{1}\right)}{n_{1}}\right)^{2}+\left(\frac{u\left(n_{2}\right)}{n_{2}}\right)^{2}}$

with $u\left(x_{1}\right)$ the uncertainty on mole fraction for component 1 and $\frac{u\left(n_{\dot{i}}\right)}{n_{i}}$ the relative uncertainty on mole number calculated from GC calibration. It should be noticed that type B uncertainty should be considered to calculate relative uncertainties on mole numbers from calibration curves. 
Table 1: Chemical samples used for experimental work.

\begin{tabular}{ccccc} 
Chemicals & $\begin{array}{c}\text { CAS } \\
\text { number }\end{array}$ & Supplier & Purity (mol \%) & $\begin{array}{c}\text { Analysis } \\
\text { method }^{\mathbf{a}}\end{array}$ \\
\hline Carbon dioxide & $124-38-9$ & Air Liquide & 99.995 & GC \\
Oxygen & $7782-44-7$ & Air Liquide & 99.999 & GC \\
\hline
\end{tabular}

${ }^{\text {a }}$ GC: Gas Chromatography

Table 2: Expected composition and real composition mole fractions.

\begin{tabular}{cccccc}
\hline $\begin{array}{c}\text { Mixture } \\
\text { number }\end{array}$ & \multicolumn{2}{c}{$\begin{array}{c}\text { Expected composition } \\
\text { mole fractions }\end{array}$} & \multicolumn{2}{c}{$\begin{array}{c}\text { Real composition } \\
\text { mole fractions }\end{array}$} & $\begin{array}{c}\text { Standard } \\
\text { uncertainties }\end{array}$ \\
& $\mathbf{C O}_{\mathbf{2}}$ & $\mathbf{O}_{\mathbf{2}}$ & $\mathbf{C O}_{\mathbf{2}}$ & $\mathbf{O}_{\mathbf{2}}$ & $\boldsymbol{u}\left(\boldsymbol{x}_{\mathbf{1}}\right)$ \\
\hline 1 & 0.7 & 0.3 & 0.726 & 0.274 & 0.003 \\
2 & 0.5 & 0.5 & 0.517 & 0.483 & 0.004 \\
3 & 0.9 & 0.1 & 0.872 & 0.128 & 0.002 \\
\hline
\end{tabular}

\subsection{Apparatus}

The Vibrating Tube Densitometer (VTD), Anton Paar DMA 512P was used to measure the densities. This equipment is similar to that described in previous work by Rivollet et al. ${ }^{9}$, Coquelet et al. ${ }^{10}$ or Nazeri et al. ${ }^{11}$. Figure 1, from Rivollet et al., presents a schematic diagram of the apparatus. 


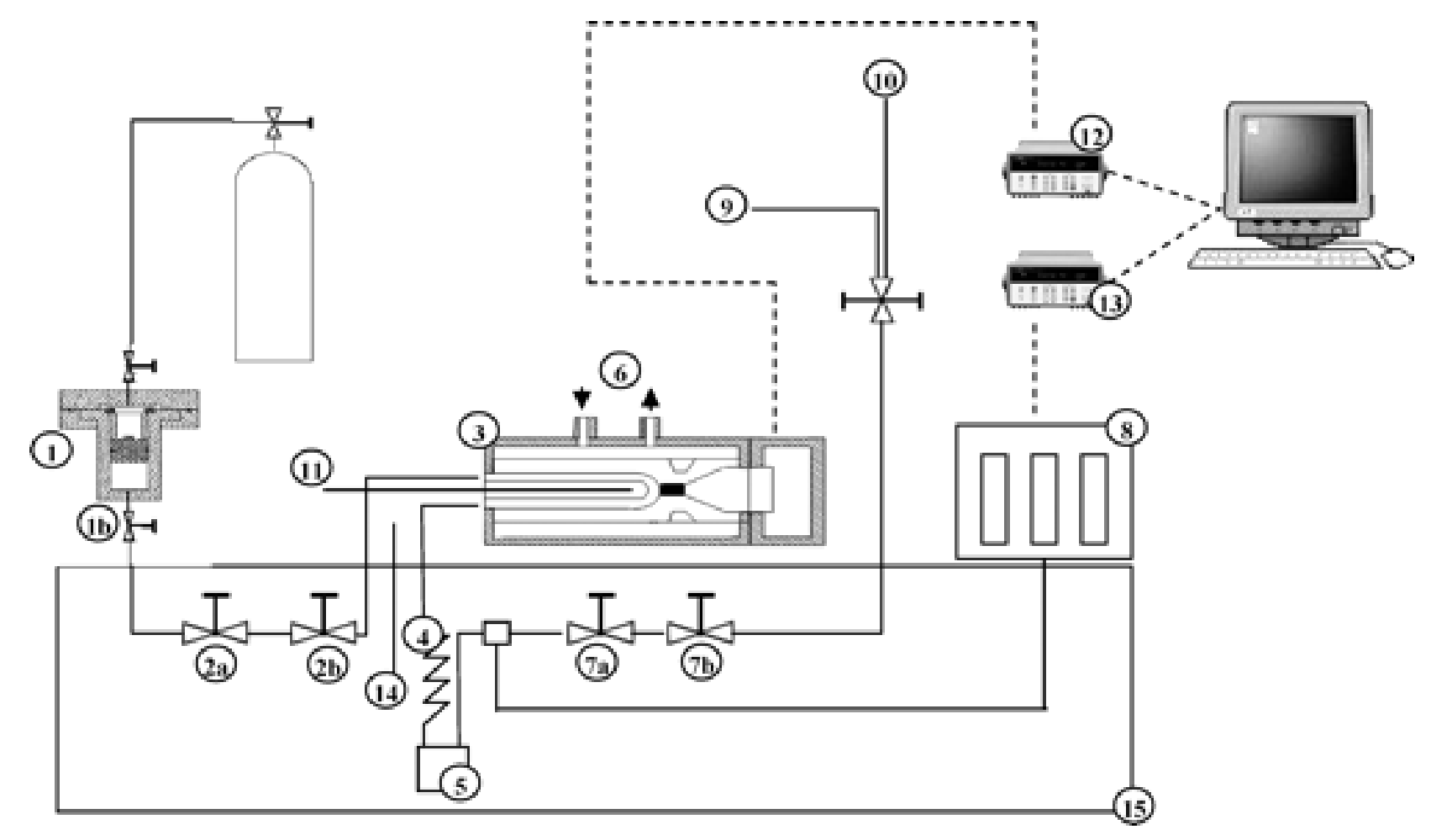

Figure 1: Flow diagram of the equipment, from Rivollet et al. ${ }^{9}:$ 1, loading cell; 2a and 2b, regulating and shut-off valves; 3, DMA 512 P densitometer (Anton Paar); 4, heat exchanger; 5, bursting disk; 6, inlet and outlet of the temperature regulating fluid; $7 \mathrm{a}$ and $7 \mathrm{~b}$, regulating and shut-off valves; 8, pressure sensors maintained at constant temperature (373 K); 9, to vacuum pump; 10, vent; 11, vibrating cell temperature sensor; 12, HP 53131A unit; 13, HP 34970A unit; 14, bath temperature sensor; 15, liquid bath. 
The main part of the setup is the U-shaped vibrating tube densitometer provided by Anton Paar. The specifications of the equipment are: pressure up to $140 \mathrm{MPa}$ and temperature between $263-473 \mathrm{~K}$. The tube material is made of Hastelloy. The temperature is controlled by fluid (silicon oil Kryo 20 from Lauda, Germany) that circulates in a jacket (small liquid bath) around the densitometer. The temperature stability is $\pm 0.02 \mathrm{~K}$.

The sample fluid is introduced from the gas reservoir into the densitometer through the tube with the diameter of $1.6 \mathrm{~mm}$ (1/16 inches) and valves 2 . The whole connection tubes are fully immersed in the temperature controlled liquid bath model West P6100. Four-wire $100-\Omega$ platinum resistance probes (Pt100) (PP) measure the temperature at each part of the equipment. The PP were calibrated against the $25-\Omega$ reference thermometer (model: Tinsley Precision Instrument with an uncertainty $u(T)=0.02 \mathrm{~K})$. The standard uncertainty of the temperature probes was estimated to be $u(T)=0.03 \mathrm{~K}$ after calibration. There are two thermostated pressure transducers (PT) of type Druck UNIK 5000 to measure different levels of pressure. PT1 can measure pressures up to $5 \mathrm{MPa}$, and PT2 can measure pressures up to $40 \mathrm{MPa}$. The transducers were calibrated using a dead weight tester (model: Desgranges \& Huot 5202S) for pressures up to $30 \mathrm{MPa}$.

The pressure transducers can measure the pressure with the standard uncertainties of $u(p)=$ $0.0003 \mathrm{MPa}$ and $u(p)=0.0005 \mathrm{MPa}$ in the ranges of $0-5 \mathrm{MPa}$ and 5-20 MPa, respectively. The pressure and the temperature were recorded using Agilent HP34970A data acquisition unit and the vibration period, $\tau$, also was recorded using a HP53131A data acquisition unit.

\subsection{VTD Calibration and Experimental procedure}

The calibration is performed using a reference fluid, $\mathrm{CO}_{2}$. The forced path mechanical calibration (FPMC) model ${ }^{12,13}$ and the density data predicted by Span and Wagner EoS with measured values of temperature and pressure, implemented in REFPROP 10.0 software ${ }^{10}$, were used to tune the unknown parameters in this model at full ranges of pressure for each measured isotherm. The measurement procedure is well described in previous publications (Coquelet et al. ${ }^{10}$, Nazeri et al. ${ }^{11}$ ). Figure 2 shows calibration results with $\mathrm{CO}_{2}$ at 276.6 and 395.19 K. The FPMC method links period of vibration, density, temperature and pressure. 


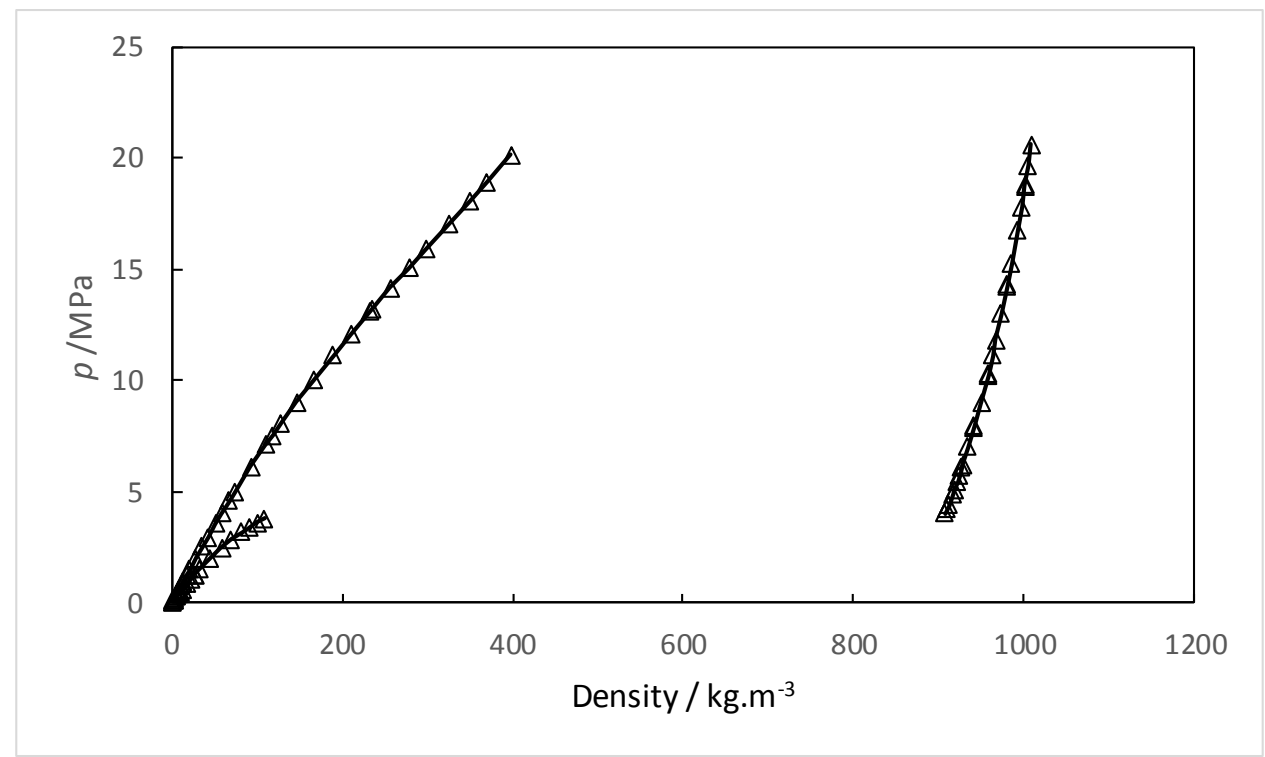

Figure 2: Calibration results with $\mathrm{CO}_{2}$ at 276.60 and 395.19K. ( $\Delta$ ): density data calculated using Span and Wagner ${ }^{15}$ model. Solid line: FPMC ${ }^{12,13}$ model.

The experimental procedure is the following. Briefly, vacuum is made in the tube. The fluid mixture is introduced as its gas phase and densities of vapor phase are measured. More fluid mixture is added in order to increase the pressure until the dew pressure. For the liquid density, fluid mixture is added until the maximum pressure (20 MPa). Density measurements are obtained by removing mixture from the VTD (decreasing pressure) until the bubble pressure. In case there is no dew or bubble pressures, starting from vacuum, the fluid mixture is added until the maximum pressure.

It is important to remind that before the appearance of dew point, the temperature of liquid bath is fixed at a value slightly higher than that of the jacket (around the vibrating tube) (difference about $0.2-0.3{ }^{\circ} \mathrm{C}$ ). It is to be sure that the first drop of fluid mixture appears exactly in the densitometer. In the same manner, before the appearance of bubble point, the temperature of liquid bath is fixed at a value slightly lower than that of the jacket. It is to be sure that the first bubble of fluid mixture appears exactly in the densitometer.

The uncertainties of densities are calculated using Eq. (2) taking into account the calibration with the reference fluid (type B) and repeatability of the measurements (acquisition of the period of vibration). Measurements are made at constant temperature and constant pressure. 
$u(\rho)=\sqrt{\left(\left(\frac{\partial \rho}{\partial p}\right)_{T, x} u(p)\right)^{2}+\left(\left(\frac{\partial \rho}{\partial T}\right)_{p, x} u(T)\right)^{2}+\left(\sum_{i}\left(\frac{\partial \rho}{\partial x_{i}}\right)_{T, p, x_{j} \neq x_{i}} u\left(x_{i}\right)\right)^{2}+\left(\frac{\alpha}{\sqrt{3}}\right)^{2}+u_{r e p}^{2}}$

where $\alpha$ represents the maximum of $\left|\rho_{\text {cal }}-\rho_{\exp }\right|$, the difference between the experimental reference fluid $\left(\mathrm{CO}_{2}\right)$ density and that calculated by the calibration curve (FPMC method) at the conditions of $T$ and $p$ and using the reference equation of state from Span and Wagner ${ }^{15}$. $\mathrm{u}_{\text {rep }}$ is the repeatability of density measurements. Temperature and pressure contributions to density uncertainty are calculated using density derivatives with respect to temperature and pressure of calibration fluid $\left(\mathrm{CO}_{2}\right)$ with the Span and Wagner ${ }^{15}$ equation of state. 


\subsection{Experimental Results}

The experimental data are presented in Tables 3 to 5 .

Table 3: Experimental isothermal density data for $\mathrm{CO}_{2}+\mathrm{O}_{2}$ binary system (Mixture 1: $\left.0.726 / 0.274\right)$ and expanded uncertainties $(k=2): U(p)=0.0003$ MPa if $P<5 \mathrm{MPa}$ and $U(p)=0.0005 \mathrm{MPa}$ if $P>5 \mathrm{MPa}$. Italic grey -shaded values correspond to possible metastable states.

\begin{tabular}{|c|c|c|c|c|c|c|c|c|c|c|c|c|c|c|c|}
\hline \multicolumn{2}{|c|}{$T=276.59 \mathrm{~K}$} & \multicolumn{2}{|c|}{$T=293.18 \mathrm{~K}$} & \multicolumn{2}{|c|}{$T=313.18 \mathrm{~K}$} & \multicolumn{2}{|c|}{$T=334.78 \mathrm{~K}$} & \multicolumn{2}{|c|}{$T=353.78 \mathrm{~K}$} & \multicolumn{2}{|c|}{$T=373.46 \mathrm{~K}$} & \multicolumn{2}{|c|}{$T=395.18 \mathrm{~K}$} & \multicolumn{2}{|c|}{$T=416.38 \mathrm{~K}$} \\
\hline \multicolumn{2}{|c|}{$U(T)=0.06 \mathrm{~K}$} & \multicolumn{2}{|c|}{$U(T)=0.06 \mathrm{~K}$} & \multicolumn{2}{|c|}{$U(T)=0.03 \mathrm{~K}$} & \multicolumn{2}{|c|}{$U(T)=0.04 \mathrm{~K}$} & \multicolumn{2}{|c|}{$U(T)=0.04 \mathrm{~K}$} & \multicolumn{2}{|c|}{$U(T)=0.01 \mathrm{~K}$} & \multicolumn{2}{|c|}{$U(T)=0.02 \mathrm{~K}$} & \multicolumn{2}{|c|}{$U(T)=0.03 \mathrm{~K}$} \\
\hline \multicolumn{2}{|c|}{$\begin{array}{l}U_{\min }(\rho)=0.2 \mathrm{~kg} \cdot \mathrm{m}^{-3} \\
U_{\max }(\rho)=8 \mathrm{~kg} \cdot \mathrm{m}^{-3}\end{array}$} & \multicolumn{2}{|c|}{$\begin{array}{l}U_{\min }(\rho)=0.5 \mathrm{~kg} \cdot \mathrm{m}^{-3} \\
U_{\max }(\rho)=15 \mathrm{~kg} \cdot \mathrm{m}^{-3}\end{array}$} & \multicolumn{2}{|c|}{$\begin{array}{l}U_{\min }(\rho)=0.4 \mathrm{~kg} \cdot \mathrm{m}^{-3} \\
U_{\max }(\rho)=13 \mathrm{~kg} \cdot \mathrm{m}^{-3}\end{array}$} & \multicolumn{2}{|c|}{$\begin{array}{c}U_{\min }(\rho)=0.1 \mathrm{~kg} \cdot \mathrm{m}^{-3} \\
U_{\max }(\rho)=3 \mathrm{~kg} \cdot \mathrm{m}^{-3}\end{array}$} & \multicolumn{2}{|c|}{$\begin{array}{c}U_{\min }(\rho)=0.1 \mathrm{~kg} \cdot \mathrm{m}^{-3} \\
U_{\max }(\rho)=3 \mathrm{~kg} \cdot \mathrm{m}^{-3}\end{array}$} & \multicolumn{2}{|c|}{$\begin{array}{c}U_{\min }(\rho)=0.1 \mathrm{~kg} \cdot \mathrm{m}^{-} \\
U_{\max }(\rho)=2 \mathrm{~kg} \cdot \mathrm{m}^{-3}\end{array}$} & \multicolumn{2}{|c|}{$\begin{array}{l}U_{\min }(\rho)=0.2 \mathrm{~kg} \cdot \mathrm{m}^{-3} \\
U_{\max }(\rho)=5 \mathrm{~kg} \cdot \mathrm{m}^{-3}\end{array}$} & \multicolumn{2}{|c|}{$\begin{array}{c}U_{\min }(\rho)=0.1 \mathrm{~kg} \cdot \mathrm{m}^{-3} \\
U_{\max }(\rho)=2.5 \mathrm{~kg} \cdot \mathrm{m} \\
3\end{array}$} \\
\hline$p / \mathrm{MPa}$ & $\rho / \mathbf{k g} \cdot \mathrm{m}^{-3}$ & $p / \mathrm{MPa}$ & $\rho / \mathbf{k g} \cdot \mathrm{m}^{-3}$ & $p / \mathrm{MPa}$ & $\underset{3}{\rho / \mathbf{k g} \cdot \mathbf{m}^{-}}$ & $p / \mathrm{MPa}$ & $\rho / \mathbf{k g} \cdot \mathbf{m}^{-3}$ & $p / \mathrm{MPa}$ & $\rho / \mathbf{k g} \cdot \mathrm{m}^{-3}$ & $p / \mathrm{MPa}$ & $\rho / \mathbf{k g} \cdot \mathbf{m}^{-3}$ & $p / \mathrm{MPa}$ & $\rho / \mathbf{k g} \cdot \mathrm{m}^{-3}$ & $p / \mathrm{MPa}$ & $\rho / \mathbf{k g} \cdot \mathbf{m}^{-3}$ \\
\hline \multicolumn{2}{|c|}{ Vapor phase } & 1.0261 & 17.9 & 1.0370 & 16.6 & 1.0254 & 15.4 & 1.0148 & 14.2 & 1.0219 & 13.6 & 1.0606 & 8.4 & 1.0645 & 12.6 \\
\hline 0.9987 & 18.6 & 1.2667 & 22.4 & 1.2480 & 20.1 & 1.3693 & 20.6 & 1.2659 & 17.9 & 1.0250 & 13.6 & 1.5114 & 13.0 & 1.2566 & 15.0 \\
\hline 1.2970 & 24.4 & 1.5059 & 26.8 & 1.5210 & 24.7 & 1.5113 & 22.8 & 1.5263 & 21.6 & 1.3410 & 17.9 & 2.0356 & 18.7 & 1.5084 & 18.0 \\
\hline 1.5079 & 29.0 & 2.0296 & 36.9 & 2.0296 & 33.4 & 2.0228 & 31.0 & 1.9712 & 28.1 & 1.3688 & 18.3 & 2.4969 & 25.5 & 2.0063 & 24.0 \\
\hline 2.0233 & 40.3 & 2.4379 & 45.1 & 2.5489 & 42.7 & 2.5291 & 39.1 & 2.4979 & 36.1 & 1.6226 & 21.7 & 3.0690 & 39.3 & 2.4614 & 29.6 \\
\hline 2.5375 & 51.2 & 3.0468 & 57.9 & 3.0567 & 52.0 & 3.1154 & 48.9 & 3.0511 & 44.3 & 1.9322 & 26.0 & 3.5236 & 45.3 & 2.9955 & 36.2 \\
\hline 3.0077 & 61.6 & 3.5789 & 69.7 & 3.4973 & 60.5 & 3.4998 & 55.4 & 3.6661 & 54.1 & 2.5360 & 34.5 & 4.0275 & 52.2 & 3.5505 & 43.1 \\
\hline 3.0136 & 61.7 & 4.2899 & 86.5 & 4.0055 & 70.5 & 4.0317 & 64.9 & 4.0456 & 60.1 & 3.1352 & 43.0 & 4.5200 & 58.8 & 4.0714 & 49.7 \\
\hline 3.5122 & 74.9 & 4.6668 & 96.0 & 4.4811 & 80.2 & 4.6096 & 75.3 & 4.4690 & 67.1 & 3.5451 & 48.9 & 5.1207 & 67.2 & 4.0949 & 50.0 \\
\hline
\end{tabular}




\begin{tabular}{|c|c|c|c|c|c|c|c|c|c|c|c|c|c|c|c|}
\hline 3.5278 & 75.4 & 5.0075 & 104.9 & 5.1405 & 94.3 & 5.0500 & 83.4 & 5.0535 & 76.8 & 3.5736 & 49.4 & 5.5301 & 72.8 & 4.4386 & 54.3 \\
\hline 4.0151 & 87.7 & 5.6455 & 122.5 & 5.5938 & 103.8 & 5.5832 & 93.6 & 5.6069 & 86.1 & 4.1283 & 57.4 & 5.5619 & 73.3 & 5.0657 & 62.4 \\
\hline 4.5495 & 103.9 & 6.1151 & 136.8 & 6.1023 & 115.6 & 6.0711 & 103.0 & 6.1721 & 95.8 & 4.5213 & 63.2 & 6.255 & 81.3 & 5.6212 & 69.7 \\
\hline 4.5577 & 104.1 & 6.6290 & 153.2 & 6.5735 & 127.0 & 6.5882 & 113.5 & 6.6370 & 103.8 & 4.9626 & 70.1 & 6.5614 & 87.5 & 6.1438 & 76.3 \\
\hline 5.0174 & 118.2 & 7.1301 & 170.3 & 7.0527 & 139.1 & 7.0710 & 123.3 & 7.1640 & 113.4 & 5.6150 & 79.9 & 7.0629 & 94.8 & 6.5506 & 81.6 \\
\hline 5.6142 & 140.2 & 7.6969 & 191.3 & 7.6326 & 154.4 & 7.5738 & 133.8 & 7.6479 & 122.3 & 6.1171 & 87.8 & 7.5466 & 101.8 & 7.1854 & 90.0 \\
\hline 5.6170 & 140.3 & 8.0761 & 206.6 & 8.1030 & 167.4 & 8.0426 & 143.8 & 8.2358 & 133.6 & 6.5974 & 95.4 & 8.0372 & 109.0 & 7.6051 & 95.6 \\
\hline 6.0855 & 159.3 & 8.5532 & 227.7 & 8.5405 & 180.1 & 8.5347 & 154.2 & 8.5141 & 139.0 & 6.9887 & 101.7 & 8.6102 & 117.8 & 8.1481 & 103.0 \\
\hline 6.5617 & 180.2 & 9.0386 & 251.3 & 9.0941 & 196.8 & 9.1248 & 168.4 & 9.1170 & 150.6 & 7.5485 & 110.7 & 9.0850 & 124.5 & 8.6379 & 109.6 \\
\hline 7.0648 & 212.1 & 9.5283 & 275.9 & 9.5589 & 211.0 & 9.5279 & 178.0 & 9.8112 & 164.2 & 8.0059 & 118.3 & 9.5294 & 131.3 & 9.1100 & 115.9 \\
\hline 7.5345 & 237.6 & 10.0108 & 301.3 & 10.0306 & 225.6 & 10.1171 & 192.3 & 10.4826 & 177.8 & 8.5713 & 127.7 & 10.0365 & 139.0 & 9.4628 & 120.7 \\
\hline 7.5454 & 239.8 & 10.4985 & 329.8 & 10.4962 & 240.7 & 10.5476 & 203.9 & 11.1379 & 191.1 & 8.5873 & 128.0 & 10.5693 & 147.2 & 10.1724 & 130.6 \\
\hline 8.762 & 276.9 & 11.0112 & 359.2 & 10.4996 & 240.9 & 10.5513 & 204.0 & 11.5980 & 200.6 & 9.0714 & 136.2 & 10.5939 & 147.6 & 10.9012 & 140.6 \\
\hline 10.7845 & 552.3 & 11.4862 & 387.6 & 10.9917 & 257.9 & 11.0054 & 214.9 & 12.1985 & 213.3 & 9.0863 & 136.4 & 10.9452 & 153.0 & 12.0581 & 156.7 \\
\hline 11.0870 & 574.2 & 11.9968 & 416.8 & 10.9961 & 258.2 & 11.5894 & 229.4 & 12.5279 & 220.3 & 9.5341 & 144.1 & 11.7240 & 165.1 & 13.1753 & 172.4 \\
\hline 11.6098 & 586.4 & 12.5082 & 444.7 & 11.4993 & 276.8 & 12.0057 & 239.9 & 12.9138 & 228.9 & 11.0376 & 170.5 & 12.7256 & 181.0 & 14.0531 & 184.8 \\
\hline \multicolumn{2}{|c|}{ Liquid phase } & 13.0310 & 471.3 & 11.9899 & 295.7 & 12.5677 & 254.2 & 13.5977 & 244.4 & 12.2323 & 192.0 & 13.6496 & 195.6 & 14.9349 & 197.3 \\
\hline 12.5564 & 632.5 & 13.0168 & 472.3 & 12.4999 & 315.7 & 12.9861 & 265.2 & 14.0973 & 254.8 & 12.2374 & 192.1 & 14.1742 & 204.0 & 16.6896 & 221.9 \\
\hline 13.5475 & 667.3 & 13.3725 & 489.3 & 12.9650 & 334.1 & 13.5110 & 279.1 & 14.1058 & 254.8 & 13.0519 & 207.0 & 14.2085 & 204.5 & 17.9826 & 240.2 \\
\hline 14.5748 & 694.3 & 14.6538 & 538.5 & 13.5010 & 354.7 & 14.0864 & 296.3 & 14.8020 & 270.2 & 14.0469 & 225.4 & 15.3271 & 222.5 & 18.9390 & 253.8 \\
\hline 15.6302 & 719.2 & 14.6416 & 542.8 & 14.0319 & 374.8 & 14.0934 & 296.7 & 15.9300 & 294.4 & 14.9435 & 242.0 & 16.1060 & 235.3 & 20.2738 & 272.8 \\
\hline 16.7981 & 741.7 & 14.9744 & 557.7 & 14.3690 & 387.6 & 14.5085 & 306.8 & 16.9908 & 318.8 & 15.3993 & 261.7 & 16.2034 & 236.9 & & \\
\hline 17.7179 & 755.6 & 16.0189 & 589.4 & 14.9869 & 408.8 & 14.9433 & 321.5 & 16.9956 & 318.9 & 17.0421 & 281.6 & 17.2424 & 253.8 & & \\
\hline 18.4966 & 767.6 & 17.0775 & 618.3 & 15.3925 & 425.7 & 15.5007 & 334.6 & 17.4785 & 331.1 & 17.9468 & 298.4 & 18.1893 & 269.1 & & \\
\hline \multirow[t]{7}{*}{20.2495} & 789.5 & 17.0630 & 619.1 & 15.6194 & 432.4 & 15.9823 & 348.6 & 18.3694 & 348.7 & 18.9235 & 316.3 & 19.2014 & 285.3 & & \\
\hline & & 18.0731 & 643.4 & 15.9551 & 443.5 & 16.4904 & 362.1 & 19.3581 & 369.9 & 20.1421 & 338.8 & 19.2265 & 285.7 & & \\
\hline & & 18.9935 & 662.1 & 16.4724 & 459.7 & 17.0442 & 376.6 & 20.4754 & 394.1 & 20.1378 & 338.8 & 20.2283 & 301.6 & & \\
\hline & & 20.2968 & 688.1 & 16.9899 & 476.0 & 17.4992 & 388.6 & & & & & & & & \\
\hline & & & & 17.5065 & 491.2 & 18.0409 & 402.6 & & & & & & & & \\
\hline & & & & 17.5148 & 491.1 & 18.5295 & 414.8 & & & & & & & & \\
\hline & & & & 17.9399 & 503.6 & 18.9942 & 426.1 & & & & & & & & \\
\hline
\end{tabular}




$\begin{array}{llll}18.4855 & 517.8 & 19.5023 & 438.6 \\ 19.0051 & 531.2 & 19.9399 & 449.1 \\ 19.4521 & 543.1 & 20.3140 & 457.3 \\ 19.4581 & 543.0 & & \\ 20.2928 & 562.5 & & \end{array}$


Table 4: Experimental isothermal density data for $\mathrm{CO}_{2}+\mathrm{O}_{2}$ binary system (mixture 2:0.517/0.483) and expanded uncertainties $(k=2): . U(p)=0.0003 \mathrm{MPa}$ if $P<5 \mathrm{MPa}$ and $U(p)=0.0005 \mathrm{MPa}$ if $P>5 \mathrm{MPa}$

\begin{tabular}{|c|c|c|c|c|c|c|c|c|c|c|c|c|c|c|c|}
\hline \multicolumn{2}{|c|}{$T=280.63 \mathrm{~K}$} & \multicolumn{2}{|c|}{$T=293.92 \mathrm{~K}$} & \multicolumn{2}{|c|}{$T=313.63 \mathrm{~K}$} & \multicolumn{2}{|c|}{$T=333.21 \mathrm{~K}$} & \multicolumn{2}{|c|}{$T=353.46 \mathrm{~K}$} & \multicolumn{2}{|c|}{$T=373.60 \mathrm{~K}$} & \multicolumn{2}{|c|}{$T=395.72 \mathrm{~K}$} & \multicolumn{2}{|c|}{$T=412.85 \mathrm{~K}$} \\
\hline \multicolumn{2}{|c|}{$U(T)=0.3 \mathrm{~K}$} & \multicolumn{2}{|c|}{$U(T)=0.2 \mathrm{~K}$} & \multicolumn{2}{|c|}{$U(T)=0.07 \mathrm{~K}$} & \multicolumn{2}{|c|}{$U(T)=0.09 \mathrm{~K}$} & \multicolumn{2}{|c|}{$U(T)=0.06 \mathrm{~K}$} & \multicolumn{2}{|c|}{$U(T)=0.1 \mathrm{~K}$} & \multicolumn{2}{|c|}{$U(T)=0.05 \mathrm{~K}$} & \multicolumn{2}{|c|}{$U(T)=0.1 \mathrm{~K}$} \\
\hline \multicolumn{2}{|c|}{$U_{\min }(\rho)=0.3 \mathrm{~kg} \cdot \mathrm{m}^{-3}$} & \multicolumn{2}{|c|}{$U_{\min }(\rho)=0.1 \mathrm{~kg} \cdot \mathrm{m}^{-3}$} & \multicolumn{2}{|c|}{$U_{\min }(\rho)=0.1 \mathrm{~kg} \cdot \mathrm{m}^{-3}$} & \multicolumn{2}{|c|}{$U_{\min }(\rho)=0.1 \mathrm{~kg} \cdot \mathrm{m}^{-3}$} & \multicolumn{2}{|c|}{$U_{\min }(\rho)=0.1 \mathrm{~kg} \cdot \mathrm{m}^{-3}$} & \multicolumn{2}{|c|}{$U_{\min }(\rho)=0.1 \mathrm{~kg} \cdot \mathrm{m}^{-3}$} & \multicolumn{2}{|c|}{$U_{\min }(\rho)=0.5 \mathrm{~kg} \cdot \mathrm{m}^{-3}$} & \multicolumn{2}{|c|}{$U_{\min }(\rho)=0.2 \mathrm{~kg} \cdot \mathrm{m}^{-3}$} \\
\hline \multicolumn{2}{|c|}{$U_{\max }(\rho)=9 \mathbf{~ k g} \cdot \mathrm{m}^{-3}$} & \multicolumn{2}{|c|}{$U_{\max }(\rho)=3 \mathrm{~kg} \cdot \mathrm{m}^{-3}$} & \multicolumn{2}{|c|}{$U_{\max }(\rho)=2.7 \mathrm{~kg} \cdot \mathrm{m}^{-3}$} & \multicolumn{2}{|c|}{$U_{\max }(\rho)=2.5 \mathrm{~kg} \cdot \mathrm{m}^{-3}$} & \multicolumn{2}{|c|}{$U_{\max }(\rho)=2 \mathrm{~kg} \cdot \mathrm{m}^{-3}$} & \multicolumn{2}{|c|}{$U_{\max }(\rho)=2 \mathrm{~kg} \cdot \mathrm{m}^{-3}$} & \multicolumn{2}{|c|}{$U_{\max }(\rho)=7 \mathrm{~kg} \cdot \mathrm{m}^{-3}$} & $U_{\max }(\rho)=$ & $4 \mathrm{~kg} \cdot \mathrm{m}^{-3}$ \\
\hline $\mathrm{p} / \mathrm{MPa}$ & $\rho / \mathbf{k g} \cdot \mathbf{m}^{-3}$ & $\mathrm{p} / \mathrm{MPa}$ & $\rho / \mathbf{k g} \cdot \mathbf{m}^{-3}$ & $p / \mathrm{MPa}$ & $\rho / \mathbf{k g} \cdot \mathbf{m}^{-3}$ & $p / \mathrm{MPa}$ & $\rho / \mathbf{k g} \cdot \mathbf{m}^{-3}$ & $\mathrm{p} / \mathrm{MPa}$ & $\rho / \mathbf{k g} \cdot \mathbf{m}^{-3}$ & $\mathrm{p} / \mathrm{MPa}$ & $\rho / \mathbf{k g} \cdot \mathbf{m}^{-3}$ & $p / \mathrm{MPa}$ & $\rho / \mathbf{k g} \cdot \mathbf{m}^{-3}$ & $\mathrm{p} / \mathrm{MPa}$ & $\rho / \mathbf{k g} \cdot \mathbf{m}^{-3}$ \\
\hline 1.0219 & 17.2 & 1.0246 & 16.3 & 1.0472 & 15.3 & 1.0155 & 14.3 & 1.0444 & 13.6 & 1.0241 & 12.6 & 1.0653 & 12.8 & 1.0441 & 11.7 \\
\hline 1.3394 & 22.9 & 1.2331 & 19.7 & 1.2643 & 18.7 & 1.2265 & 17.3 & 1.2764 & 16.8 & 1.2614 & 15.6 & 1.2743 & 15.3 & 1.2864 & 14.5 \\
\hline 1.6537 & 28.7 & 1.5047 & 24.3 & 1.5056 & 22.4 & 1.5333 & 21.6 & 1.5315 & 20.2 & 1.5458 & 19.2 & 1.5488 & 18.6 & 1.5657 & 17.4 \\
\hline 2.0153 & 35.2 & 2.0222 & 33.0 & 2.0467 & 30.8 & 2.0282 & 28.9 & 2.0277 & 26.9 & 2.0885 & 26.0 & 2.0313 & 24.5 & 2.0840 & 23.4 \\
\hline 2.5069 & 44.6 & 2.5310 & 41.9 & 2.6601 & 40.5 & 2.5609 & 36.6 & 2.5469 & 34.0 & 2.5646 & 32.0 & 2.5585 & 31.0 & 2.5089 & 28.1 \\
\hline 3.0256 & 54.6 & 3.0318 & 50.9 & 2.6690 & 40.6 & 3.0575 & 43.9 & 3.0411 & 40.8 & 3.5445 & 44.8 & 3.0501 & 37.0 & 3.0522 & 34.3 \\
\hline 3.5492 & 64.9 & 3.5266 & 60.0 & 3.0530 & 46.8 & 3.5308 & 51.1 & 3.5362 & 47.7 & 4.0720 & 51.8 & 3.5160 & 42.8 & 3.5102 & 39.7 \\
\hline 4.0264 & 75.1 & 4.1454 & 71.7 & 3.5421 & 54.9 & 4.0576 & 59.4 & 4.0019 & 54.3 & 4.5326 & 57.8 & 4.0235 & 49.1 & 3.5129 & 39.7 \\
\hline 4.5352 & 86.3 & 4.7065 & 82.7 & 4.0055 & 62.7 & 4.5343 & 67.0 & 4.5310 & 61.9 & 5.0571 & 64.8 & 4.5348 & 55.6 & 4.0658 & 46.1 \\
\hline 5.0437 & 97.6 & 5.2948 & 94.6 & 4.5119 & 71.3 & 5.0226 & 75.5 & 5.0639 & 69.6 & 5.6110 & 72.2 & 5.0142 & 61.5 & 4.5342 & 51.5 \\
\hline 5.5936 & 110.5 & 6.1200 & 112.1 & 5.0869 & 81.4 & 5.6148 & 85.6 & 5.6206 & 77.7 & 6.5922 & 85.5 & 5.6212 & 69.4 & 5.0091 & 57.1 \\
\hline 6.0780 & 122.1 & 6.8166 & 127.4 & 5.5060 & 88.9 & 6.1091 & 93.1 & 6.0908 & 84.7 & 7.0975 & 92.4 & 6.1170 & 75.7 & 5.5898 & 63.9 \\
\hline 6.5663 & 134.3 & 7.2607 & 137.5 & 6.1028 & 99.8 & 6.5675 & 100.4 & 6.6584 & 93.2 & 7.5801 & 99.0 & 6.5478 & 81.2 & 6.1112 & 70.1 \\
\hline 6.9954 & 145.9 & 8.0523 & 156.1 & 6.1485 & 100.6 & 7.1151 & 109.5 & 7.1090 & 100.1 & 8.0887 & 106.1 & 7.1777 & 89.4 & 6.1177 & 70.2 \\
\hline 7.5527 & 161.2 & 8.5833 & 169.2 & 6.1635 & 100.9 & 7.5868 & 117.5 & 7.6165 & 107.9 & 8.5637 & 112.8 & 7.5727 & 94.5 & 6.5145 & 74.8 \\
\hline 8.0253 & 174.1 & 9.4241 & 190.6 & 6.5114 & 107.3 & 8.0747 & 126.1 & 8.1279 & 115.6 & 9.0477 & 119.6 & 8.0988 & 101.3 & 6.5351 & 75.1 \\
\hline
\end{tabular}




\begin{tabular}{|c|c|c|c|c|c|c|c|c|c|c|c|c|c|c|c|}
\hline 8.6304 & 192.0 & 10.1205 & 208.9 & 7.1026 & 118.6 & 8.5662 & 134.7 & 8.5896 & 122.8 & 9.5688 & 126.9 & 8.5463 & 107.2 & 7.0876 & 81.6 \\
\hline 8.6246 & 192.2 & 11.5615 & 248.2 & 7.1133 & 118.8 & 9.0788 & 143.9 & 9.1311 & 131.4 & 10.0544 & 133.9 & 9.0976 & 114.3 & 7.5998 & 87.8 \\
\hline 9.0663 & 205.8 & 11.5565 & 248.4 & 7.6008 & 128.2 & 9.5489 & 152.3 & 10.0255 & 145.6 & 11.0624 & 148.2 & 9.5775 & 120.7 & 8.0564 & 93.3 \\
\hline 9.6689 & 224.2 & 12.0924 & 263.5 & 8.1760 & 139.8 & 10.0527 & 161.3 & 11.0868 & 162.4 & 12.0623 & 162.5 & 10.1030 & 127.6 & 8.5614 & 99.3 \\
\hline 10.0351 & 235.6 & 12.0880 & 263.5 & 8.2646 & 141.6 & 10.5497 & 170.4 & 12.0187 & 177.5 & 13.0453 & 176.6 & 11.0325 & 140.0 & 8.5748 & 99.4 \\
\hline 11.0633 & 269.8 & 13.2030 & 295.6 & 9.5421 & 167.7 & 11.0536 & 179.6 & 13.0730 & 194.8 & 13.9880 & 190.2 & 12.1180 & 154.3 & 9.0691 & 105.4 \\
\hline 11.0517 & 270.4 & 13.1990 & 295.9 & 10.0591 & 178.4 & 11.5643 & 189.0 & 13.0725 & 194.8 & 14.8730 & 202.6 & 13.0650 & 167.0 & 9.5479 & 111.2 \\
\hline 11.9929 & 303.1 & 14.0458 & 319.4 & 11.0860 & 200.7 & 12.0412 & 198.5 & 14.0107 & 210.2 & 16.0800 & 220.5 & 14.0217 & 179.8 & 10.0528 & 117.3 \\
\hline 13.0339 & 340.0 & 14.0378 & 319.5 & 12.1605 & 224.4 & 12.5159 & 207.7 & 15.0277 & 226.9 & 16.0822 & 220.6 & 15.1979 & 195.4 & 10.8987 & 127.6 \\
\hline 14.0656 & 376.6 & 15.0380 & 348.4 & 13.0074 & 243.4 & 13.0458 & 217.8 & 15.9847 & 243.0 & 17.0522 & 235.1 & 16.2579 & 209.6 & 12.1664 & 142.7 \\
\hline 15.0294 & 410.6 & 15.0341 & 348.7 & 14.316 & 271.7 & 13.5555 & 227.5 & 17.0015 & 259.8 & 17.0555 & 235.1 & 17.0434 & 220.2 & 13.0663 & 153.5 \\
\hline 16.2689 & 449.0 & 16.6414 & 392.3 & 14.9472 & 288.2 & 13.9996 & 236.0 & 18.0245 & 276.5 & 17.8714 & 247.1 & 18.1289 & 234.7 & 14.1484 & 166.9 \\
\hline 16.2619 & 449.5 & 16.6354 & 392.7 & 15.9882 & 311.6 & 14.5189 & 245.6 & 18.9421 & 291.1 & 19.0095 & 263.6 & 19.3526 & 250.7 & 16.4731 & 195.5 \\
\hline 16.9518 & 468.7 & 17.9488 & 426.4 & 16.1772 & 315.8 & 15.0036 & 254.7 & 20.3158 & 312.6 & 19.0090 & 263.6 & 20.2032 & 261.7 & 17.0456 & 202.3 \\
\hline 16.9476 & 468.9 & 17.9420 & 426.8 & 17.4641 & 344.8 & 15.9818 & 273.4 & & & 20.0154 & 277.9 & & & 18.4366 & 218.7 \\
\hline 18.5980 & 510.7 & 19.1108 & 456.3 & 18.8837 & 375.4 & 16.9748 & 292.6 & & & & & & & 20.0959 & 238.3 \\
\hline 18.5864 & 511.2 & 20.1976 & 480.4 & 20.1692 & 401.9 & 18.0141 & 312.7 & & & & & & & & \\
\hline \multirow[t]{3}{*}{20.3276} & 553.1 & & & 20.1674 & 401.9 & 19.0085 & 331.1 & & & & & & & & \\
\hline & & & & & & 19.9326 & 348.1 & & & & & & & & \\
\hline & & & & & & 20.2304 & 353.8 & & & & & & & & \\
\hline
\end{tabular}


Table 5: Experimental isothermal density data for $\mathrm{CO}_{2}+\mathrm{O}_{2}$ binary system (mixture 3: 0.872/0.128) and expanded uncertainties $(k=2): U(p)=0.0003 \mathrm{MPa}$ if $P<5 \mathrm{MPa}$ and $U(p)=0.0005 \mathrm{MPa}$ if $P>5 \mathrm{MPa}$. Italic grey -shaded values correspond to possible metastable states.

\begin{tabular}{|c|c|c|c|c|c|c|c|c|c|c|c|c|c|c|c|}
\hline \multicolumn{2}{|c|}{$T=279.05 \mathrm{~K}$} & \multicolumn{2}{|c|}{$T=293.31 \mathrm{~K}$} & \multicolumn{2}{|c|}{$T=315.06 \mathrm{~K}$} & \multicolumn{2}{|c|}{$T=333.25 \mathrm{~K}$} & \multicolumn{2}{|c|}{$T=353.62 \mathrm{~K}$} & \multicolumn{2}{|c|}{$T=374.23 \mathrm{~K}$} & \multicolumn{2}{|c|}{$T=394.15 \mathrm{~K}$} & \multicolumn{2}{|c|}{$T=414.31 \mathrm{~K}$} \\
\hline \multicolumn{2}{|c|}{$U(T)=0.1 \mathrm{~K}$} & \multicolumn{2}{|c|}{$U(T)=0.09 \mathrm{~K}$} & \multicolumn{2}{|c|}{$U(T)=0.07 \mathrm{~K}$} & \multicolumn{2}{|c|}{$U(T)=0.04 \mathrm{~K}$} & \multicolumn{2}{|c|}{$U(T)=0.03 \mathrm{~K}$} & \multicolumn{2}{|c|}{$U(T)=0.07 \mathrm{~K}$} & \multicolumn{2}{|c|}{$U(T)=0.03 \mathrm{~K}$} & \multicolumn{2}{|c|}{$U(T)=0.02 \mathrm{~K}$} \\
\hline \multicolumn{2}{|c|}{$\operatorname{Umin}(\rho)=0.3 \mathrm{~kg} \cdot \mathrm{m}^{-3}$} & \multicolumn{2}{|c|}{$\operatorname{Umin}(\rho)=0.2 \mathrm{~kg} \cdot \mathrm{m}^{-3}$} & \multicolumn{2}{|c|}{$\operatorname{Umin}(\rho)=0.1 \mathrm{~kg} \cdot \mathrm{m}^{-3}$} & \multicolumn{2}{|c|}{$\begin{array}{c}\operatorname{Umin}(\rho)=0.1 \\
\mathrm{~kg} \cdot \mathrm{m}^{-3}\end{array}$} & \multicolumn{2}{|c|}{$\begin{array}{c}U \min (\rho)=0.1 \\
k g \cdot m^{-3}\end{array}$} & \multicolumn{2}{|c|}{$\begin{array}{c}U \min (\rho)=0.4 \\
\mathrm{~kg} \cdot \mathrm{m}^{-3}\end{array}$} & \multicolumn{2}{|c|}{$\begin{array}{c}U \min (\rho)=0.1 \\
\mathrm{~kg} \cdot \mathrm{m}^{-3}\end{array}$} & \multicolumn{2}{|c|}{$\begin{array}{l}U \min (\rho)= \\
0.1 \mathrm{~kg} \cdot \mathrm{m}^{-3}\end{array}$} \\
\hline \multicolumn{2}{|c|}{$U \max (\rho)=13 \mathrm{~kg} \cdot \mathrm{m}^{-3}$} & \multicolumn{2}{|c|}{$U \max (\rho)=9 \mathrm{~kg} \cdot \mathrm{m}^{3}$} & \multicolumn{2}{|c|}{$U \max (\rho)=4 \mathrm{~kg} \cdot \mathrm{m}^{-3}$} & \multicolumn{2}{|c|}{$U \max (\rho)=4 \mathrm{~kg} \cdot \mathrm{m}^{-3}$} & \multicolumn{2}{|c|}{$\operatorname{Umax}_{3}(\rho)=3 \mathrm{~kg} \cdot \mathrm{m}^{-}$} & \multicolumn{2}{|c|}{$\underset{3}{U \max }(\rho)=4 \mathrm{~kg} \cdot \mathrm{m}^{-}$} & \multicolumn{2}{|c|}{$\begin{array}{c}U \max (\rho)=2.5 \\
\operatorname{kg} \cdot \mathrm{m}^{-3}\end{array}$} & \multicolumn{2}{|c|}{$\begin{array}{c}U \max (\rho)=2.5 \\
\text { kg. } \mathrm{m}^{-3}\end{array}$} \\
\hline$p / \mathrm{MPa}$ & $\rho / \mathbf{k g} \cdot \mathbf{m}^{-3}$ & $p / M P a$ & $\rho /$ kg.m ${ }^{-3}$ & $\mathrm{p} / \mathrm{MPa}$ & $\underset{3}{\rho / \mathbf{k g} \cdot \mathbf{m}^{-}}$ & $\mathrm{p} / \mathrm{MPa}$ & $\rho_{3}^{\rho / k g . m^{-}}$ & $\mathrm{p} / \mathrm{MPa}$ & $\underset{3}{\rho / \mathbf{k g} \cdot \mathbf{m}^{-}}$ & $\mathrm{p} / \mathrm{MPa}$ & $\underset{3}{\rho / \mathbf{k g}^{-\mathbf{m}^{-}}}$ & $p / \mathrm{MPa}$ & $\underset{3}{\rho / \mathbf{k g} \cdot \mathbf{m}^{-}}$ & $p / \mathrm{MPa}$ & $\underset{3}{\rho / \mathrm{kg} \cdot \mathrm{m}}$ \\
\hline Vap & lase & Vap & ase & 1.0407 & 17.5 & 1.0219 & 16.2 & 1.0073 & 14.9 & 1.0273 & 14.5 & 1.0665 & 14.1 & 1.0140 & 12.7 \\
\hline 1.0416 & 20.4 & 1.0043 & 18.6 & 1.2437 & 21.0 & 1.2800 & 20.4 & 1.5203 & 22.9 & 1.5180 & 21.6 & 1.2007 & 15.8 & 1.5137 & 19.1 \\
\hline 1.2633 & 25.1 & 1.2769 & 24.0 & 1.5758 & 27.0 & 1.5362 & 24.7 & 2.0373 & 31.1 & 2.0810 & 29.9 & 1.4843 & 19.7 & 2.0358 & 25.9 \\
\hline 1.5048 & 30.5 & 1.5412 & 29.3 & 1.9996 & 34.9 & 2.0297 & 33.1 & 2.5328 & 39.1 & 2.5194 & 36.5 & 2.0318 & 27.1 & 2.5355 & 32.4 \\
\hline 2.0163 & 42.2 & 2.0404 & 40.0 & 2.5358 & 45.3 & 2.5422 & 42.2 & 3.1299 & 49.2 & 2.9868 & 43.8 & 2.4654 & 33.2 & 3.0494 & 39.3 \\
\hline 2.5130 & 53.7 & 2.5863 & 52.2 & 3.0392 & 55.4 & 3.0561 & 51.7 & 3.5407 & 56.2 & 3.5699 & 53.1 & 3.0207 & 41.0 & 3.5748 & 46.3 \\
\hline 3.0151 & 68.2 & 3.0629 & 63.6 & 3.5599 & 66.4 & 3.5237 & 60.6 & 4.0393 & 65.0 & 4.0447 & 61.9 & 3.5575 & 48.8 & 4.0597 & 52.9 \\
\hline 3.2625 & 76.1 & 3.5193 & 75.2 & 4.0532 & 77.6 & 4.0380 & 70.8 & 4.5088 & 73.5 & 4.5060 & 71.0 & 3.9505 & 54.6 & 4.0584 & 52.9 \\
\hline 3.5074 & 84.8 & 4.0205 & 89.6 & 4.5274 & 88.9 & 4.6668 & 83.8 & 5.0562 & 83.8 & 5.0295 & 78.5 & 4.5499 & 63.5 & 4.5645 & 60.0 \\
\hline 3.7587 & 92.8 & 4.5284 & 104.9 & 5.1645 & 105.1 & 5.0349 & 91.7 & 5.7330 & 97.0 & 5.5493 & 86.4 & 5.0643 & 71.3 & 5.0419 & 66.6 \\
\hline 4.1541 & 106.1 & 5.0349 & 122.0 & 5.5868 & 116.7 & 5.5930 & 104.3 & 6.0705 & 103.1 & 6.1195 & 96.4 & 6.0782 & 87.1 & 5.6518 & 75.3 \\
\hline 4.2843 & 110.7 & 5.6098 & 144.6 & 6.1369 & 132.4 & 6.1099 & 116.4 & 6.6522 & 115.1 & 6.1232 & 96.4 & 6.5320 & 94.3 & 6.0878 & 81.6 \\
\hline 4.4095 & 115.6 & 6.0892 & 166.3 & 6.6193 & 147.5 & 6.6518 & 129.8 & 7.0612 & 124.4 & 6.6065 & 105.2 & 7.0752 & 103.1 & 6.7675 & 91.3 \\
\hline
\end{tabular}




\begin{tabular}{|c|c|c|c|c|c|c|c|c|c|c|c|c|c|c|c|}
\hline 4.5645 & 122.5 & 6.6721 & 198.5 & 7.0621 & 162.4 & 6.6534 & 129.8 & 7.5756 & 136.4 & 7.0261 & 112.9 & 7.5136 & 110.4 & 8.1022 & 111.2 \\
\hline 4.6561 & 127.0 & 6.9584 & 217.6 & 7.5584 & 180.4 & 7.0752 & 140.7 & 7.9620 & 145.4 & 7.5889 & 123.3 & 8.0078 & 118.6 & 8.4669 & 116.9 \\
\hline 4.7489 & 131.5 & 7.2267 & 240.7 & 8.0728 & 200.8 & 7.0789 & 140.8 & 8.5421 & 157.1 & 8.0379 & 131.8 & 8.0843 & 119.9 & 9.2081 & 128.4 \\
\hline 4.9287 & 143.3 & 7.3480 & 253.3 & 8.0775 & 200.9 & 7.5828 & 154.5 & 9.0142 & 166.6 & 8.5729 & 142.1 & 8.4884 & 126.8 & 10.2145 & 143.4 \\
\hline 5.0117 & 148.8 & 7.4496 & 267.7 & 8.5480 & 221.5 & 8.0965 & 168.9 & 9.5589 & 179.7 & 9.0370 & 151.4 & 8.5278 & 127.4 & 11.2972 & 161.0 \\
\hline 5.1070 & 157.2 & 7.5519 & 288.0 & 9.0717 & 247.1 & 8.5662 & 183.1 & 10.0558 & 192.0 & 10.0876 & 173.0 & 8.9956 & 135.5 & 11.9586 & 171.9 \\
\hline 5.2149 & 167.2 & 7.6842 & 316.2 & 9.5412 & 272.3 & 8.5678 & 183.2 & 10.7222 & 210.8 & 10.4917 & 181.5 & 9.0607 & 136.6 & 12.9289 & 189.1 \\
\hline 5.3002 & 172.7 & 7.8201 & 322.8 & 9.9982 & 298.6 & 9.0560 & 198.4 & 11.4360 & 232.6 & 11.0366 & 193.1 & 9.4928 & 144.2 & 13.8057 & 203.8 \\
\hline 5.3906 & 177.8 & 7.9261 & 335.4 & 10.2212 & 315.2 & 9.2307 & 203.9 & 12.0510 & 252.9 & 11.9161 & 212.7 & 9.5248 & 145.1 & 14.7433 & 219.5 \\
\hline 5.4920 & 186.0 & 8.0564 & 347.0 & 10.6028 & 335.6 & 9.5275 & 214.1 & 12.7049 & 273.9 & 12.9049 & 235.1 & 10.0376 & 154.1 & 16.1194 & 242.5 \\
\hline 5.5824 & 200.7 & 8.2371 & 389.9 & 10.6898 & 347.8 & 9.5288 & 214.2 & 13.5401 & 294.5 & 14.0828 & 262.8 & 10.5522 & 163.4 & 17.5173 & 266.3 \\
\hline 5.7402 & 223.4 & 8.3445 & 407.1 & 11.1620 & 372.0 & 9.7922 & 223.3 & 13.9400 & 304.6 & 14.9868 & 284.5 & 11.0513 & 172.6 & 18.9761 & 290.9 \\
\hline 5.8086 & 225.2 & 8.4536 & 419.9 & 11.7107 & 412.0 & 9.7998 & 223.5 & 13.9358 & 304.8 & 16.0138 & 309.0 & 11.6063 & 182.9 & 20.0204 & 308.6 \\
\hline 5.9850 & 591.8 & 8.5411 & 438.2 & 12.0508 & 435.8 & 10.0180 & 231.2 & 14.9049 & 330.9 & 16.9216 & 330.6 & 12.1181 & 192.6 & & \\
\hline 6.1056 & 613.0 & 8.6407 & 465.0 & 12.4275 & 465.9 & 10.2597 & 239.7 & 16.1022 & 367.7 & 16.9188 & 330.8 & 12.5181 & 200.2 & & \\
\hline 6.5946 & 698.3 & 8.7582 & 506.2 & 12.8458 & 491.0 & 10.5548 & 250.6 & 16.9052 & 393.3 & 18.0110 & 356.3 & 12.9645 & 208.8 & & \\
\hline 7.1041 & 712.4 & 8.9342 & 527.1 & 13.5490 & 532.0 & 11.0228 & 268.2 & 18.2172 & 432.5 & 18.8901 & 376.3 & 14.1039 & 231.0 & & \\
\hline 7.9046 & 760.2 & 9.5655 & 9.1884 & 553.1 & 558.4 & 11.4899 & 287.1 & 18.1943 & 433.4 & 18.8743 & 376.5 & 15.0024 & 248.1 & & \\
\hline 7.9152 & 760.7 & 9.5655 & 590.2 & 15.1136 & 593.3 & 12.0560 & 309.8 & 18.9987 & 458.0 & 20.0624 & 403.0 & 15.0023 & 248.1 & & \\
\hline 8.9302 & 786.0 & 11.0317 & 680.8 & 18.1689 & 677.6 & 13.4962 & 375.2 & & & & & 18.6716 & 320.1 & & \\
\hline 9.5588 & 798.3 & 12.0270 & 716.9 & 19.2425 & 697.4 & 13.9740 & 391.5 & & & & & 20.2201 & 350.2 & & \\
\hline 9.9407 & 807.3 & 12.8227 & 737.6 & 20.4052 & 716.7 & 14.4890 & 418.9 & & & & & 20.2215 & 350.4 & & \\
\hline 10.9404 & 823.2 & 13.8256 & 758.5 & & & 15.2235 & 444.0 & & & & & & & & \\
\hline 12.1374 & 838.5 & 15.0309 & 779.0 & & & 15.6111 & 459.6 & & & & & & & & \\
\hline 12.6650 & 843.9 & 15.0396 & 779.0 & & & 16.1478 & 480.1 & & & & & & & & \\
\hline 13.0291 & 850.0 & 16.0513 & 794.6 & & & 16.8926 & 505.5 & & & & & & & & \\
\hline 14.5011 & 864.8 & 16.7640 & 803.4 & & & 17.9419 & 539.0 & & & & & & & & \\
\hline 15.4452 & 873.2 & 18.0263 & 818.7 & & & 18.9661 & 569.3 & & & & & & & & \\
\hline 16.8885 & 885.7 & 18.4149 & 822.9 & & & 20.2278 & 600.3 & & & & & & & & \\
\hline
\end{tabular}


17.9250

17.9341

19.0671

19.8930

20.3845
19.4981

20.1334

20.1539

833.5

838.0

837.1
902.2

906.4

910.7 


\section{Correlation and discussions}

\subsection{Development of PR EoS}

We have used a cubic equation of state to compare its density predictions with the experimental data. Cubic equations of state are often used for the design of underground gas reservoirs as they are very easy to solve. The critical temperatures $\left(T_{c}\right)$ and pressures $\left(P_{c}\right)$ and acentric factors $(\omega)$ for pure $\mathrm{CO}_{2}$ and $\mathrm{O}_{2}$, which are collected from Simulis thermodynamic software (from Prosim, Toulouse, France), are provided in Table 6.

Table 6. Critical properties and acentric factors for Carbone dioxide and Oxygen pure components (Source Simulis thermodynamic software)

\begin{tabular}{cccc}
\hline Component & $\mathrm{T}_{\mathrm{c}} / \mathbf{K}$ & $\mathbf{P}_{\mathrm{c}} /$ bar & Acentric factor $\boldsymbol{\omega}$ \\
\hline $\mathrm{CO}_{2}$ & 304.21 & 73.83 & 0.223621 \\
$\mathrm{O}_{2}$ & 154.58 & 50.43 & 0.0221798 \\
\hline
\end{tabular}

In order to have the best representation of the phase diagrams, we have considered the Peng-Robinson Equation of State ${ }^{16}$ (Eq. 3) with the Wong Sandler Mixing rules ${ }^{17}$ (Eqs. 4 and 5) involving the NRTL activity coefficient model $^{18}$ (Eq. 6). Indeed, as $\mathrm{O}_{2}$ is a cryogenic component, the phase diagram has the particularity to have a mixture critical point. Cubic EoS have some difficulties to represent accurately the equilibrium properties close to the mixture critical point.

$$
\begin{gathered}
\left(P+\frac{a \alpha(T)}{v^{2}+2 v b-b^{2}}\right)(v-b)=R T \\
b=\frac{\sum_{i} \sum_{j}\left(b-\frac{a}{R T}\right)_{i j}}{1-\left(\frac{\sum_{i} x_{i} \frac{a_{i}}{b_{i}}}{R T}+\frac{g_{\gamma}^{E}}{C R T}\right)}
\end{gathered}
$$

with $C=\ln (1 / 2)$ 
$b-\frac{a}{R T}=\sum_{i} \sum_{j} x_{i} x_{j}\left(b-\frac{a}{R T}\right)_{i j}$ with $\left(b-\frac{a}{R T}\right)_{i j}=\frac{1}{2}\left[\left(b-\frac{a}{R T}\right)_{i}+\left(b-\frac{a}{R T}\right)_{j}\right]\left(1-k_{i j}\right)$

$g_{V}^{E}=\sum_{i} x_{i} \sum_{j} \frac{x_{j} \exp \left(-\alpha_{i j} \frac{C_{j i}}{R T}\right)}{\sum_{k} \exp \left(-\alpha_{i k} \frac{C_{k i}}{R T}\right)} C_{j i}$

with $C_{i i}=0$. The value of the non-randomness parameters, $\alpha_{\mathrm{ij}}$, is equal to 0.1 . Note that $\alpha_{i j}$ is different from $\alpha(T)$ which is the alpha function of the PR EoS.

In order to adjust the binary interaction parameters $\left(C_{i j}\right.$ and $\left.k_{i j}\right)$, we have considered a database of six references presented in Table 7. As all the systems present a mixture critical point, we have just considered an objective function of the bubble pressure (Eq. 7).

Fobj $=\sum_{i}^{N d a t a}\left(\frac{p_{\exp }-p_{\text {cal }}}{p_{\exp }}\right)^{2}$

All the data were used to fit the binary interaction parameters. We did not consider any temperature dependency of each binary interaction parameter. The obtained values are $C_{12}=479 \mathrm{~J} \cdot \mathrm{mol}^{-1}, C_{21}=-216 \mathrm{~J} \cdot \mathrm{mol}^{-1}$ and $k_{12}=0.2078$. The performance of the model was assessed by means of the following relative deviations, $A A D U$ and $B I A S U$, which are expressed as:

$A A D U=\frac{1}{N} \sum_{i=1}^{N} \frac{\left|U_{i, e x p}-U_{i, c a l}\right|}{U_{i, e x p}}$

BIAS $U=\frac{1}{N} \sum_{i=1}^{N} \frac{\left(U_{i, \exp }-U_{i, c a l}\right)}{U_{i, \exp }}$

where $U$ is the pressure ( $p$ ) or the vapor composition $\left(y_{1}\right)$ and $N$ is the number of experimental measurements. Results are presented in Table 8 and Figure 3. As can be seen, when the temperature approaches the critical temperature of $\mathrm{CO}_{2}$, the model has some difficulties to represent the equilibrium properties. In order to improve the prediction of the phase diagram, we can consider temperature dependent binary interaction parameters, but it is not judicious for density prediction at temperatures higher than the critical temperature of $\mathrm{CO}_{2}$. 
Table 7 : Summary of vapor liquid equilibrium data of the binary system $\mathrm{CO}_{2}+\mathrm{O}_{2}$.

\begin{tabular}{ccc}
\hline Reference & Type of Data & Temperatures \\
\hline Fredenslund and Sather $1970^{19}$ & $p T x y$ & $263.15,273.15$ and 283.15 \\
Kaminishi and Toriumi $1966^{20}$ & $p T x y$ & $253.15,273.15,288.15$ and 298.15 \\
Muirbrook and Prausnitz $1965^{21}$ & $p T x y$ & 273.15 \\
Zenner and Dana $1963^{22}$ & $p T x y$ & 273.15 \\
Lasala et al. $2016^{23}$ & $p T x y$ & $273.15,288.15$ and 298.15 \\
Westman et al. $2016^{24}$ & $p T x y$ & 273.09 and 293.08 \\
\hline
\end{tabular}

Table 8: Values of $B I A S$, and $A A D$ of pressure and vapor compositions for the different sets of data.

\begin{tabular}{|c|c|c|c|c|c|}
\hline Reference & $T / K$ & BIAS $p / \%$ & BIAS y/\% & $A A D p / \%$ & $A A D$ y $/ \%$ \\
\hline \multirow{3}{*}{ Fredenslund and Sather $1970^{19}$} & 263.15 & 6.1 & -1.6 & 6.1 & 2.5 \\
\hline & 273.15 & 4.8 & -1.7 & 4.8 & 2.3 \\
\hline & 283.15 & 8.5 & -3.7 & 8.5 & 3.7 \\
\hline \multirow{4}{*}{ Kaminishi and Toriumi $1966^{20}$} & 253.15 & 6.9 & -7.6 & 7.8 & 7.6 \\
\hline & 273.15 & 6.3 & -4.4 & 6.3 & 4.4 \\
\hline & 288.15 & 5.6 & -4.2 & 5.6 & 4.2 \\
\hline & 298.15 & 3.5 & -2.0 & 3.5 & 2.0 \\
\hline Muirbrook and Prausnitz $1965^{21}$ & 273.15 & 5.7 & -1.8 & 5.7 & 3.4 \\
\hline Zenner and Dana $1963^{22}$ & 273.15 & 4.6 & -4.5 & 5.8 & 4.7 \\
\hline \multirow{3}{*}{ Lasala et al. $2016^{23}$} & 273.15 & 12.6 & -3.8 & 12.6 & 3.8 \\
\hline & 288.15 & 6.4 & -2.8 & 6.4 & 2.8 \\
\hline & 298.15 & \multicolumn{4}{|c|}{ Calculation failed close to mixture critical point } \\
\hline \multirow{2}{*}{ Westman et al. $2016^{24}$} & 273.09 & 9.8 & -3.3 & 9.8 & 3.9 \\
\hline & 293.08 & 2.1 & -1.2 & 2.1 & 1.2 \\
\hline
\end{tabular}




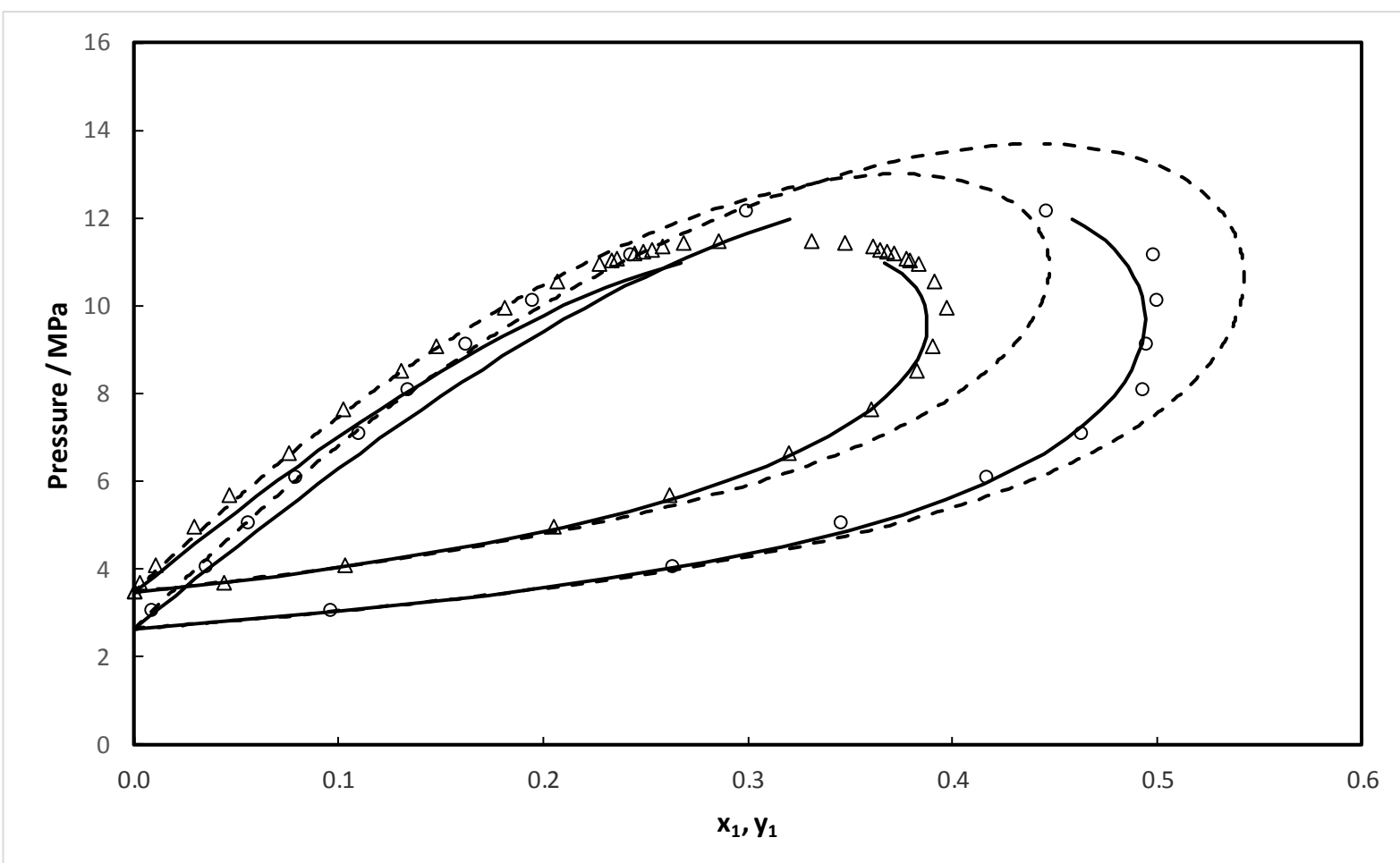

Figure 3 : Vapor - Liquid equilibrium isotherms for $\mathrm{O}_{2}(1)+\mathrm{CO}_{2}(2)$ binary system: $(\Delta)$ : $273.09 \mathrm{~K}$ from Westman et al. ${ }^{24}$, (o), 263.15 K from Fredenslund and Sather ${ }^{19}$. Solid line: Peng Robinson EoS model, Dashed line: EoS-CG (Gernert and $\operatorname{Span}^{8}$ ) EoS model.

$P T$ envelopes of the three mixtures (see Table 2) are predicted using our thermodynamic model and plotted in Figure 4. In this figure, we have also plotted the PT data of each system corresponding to the measured densities. According to Figure 4, we can observe that few density data were determined in the vapor-liquid region. Probably for these data, we are in a metastable state (mixture 3, $T=279.05$ and $293.31 \mathrm{~K}$, mixture 1, $T=276.59 \mathrm{~K}$ ). These data are mentioned in Tables 3 and 5 . 


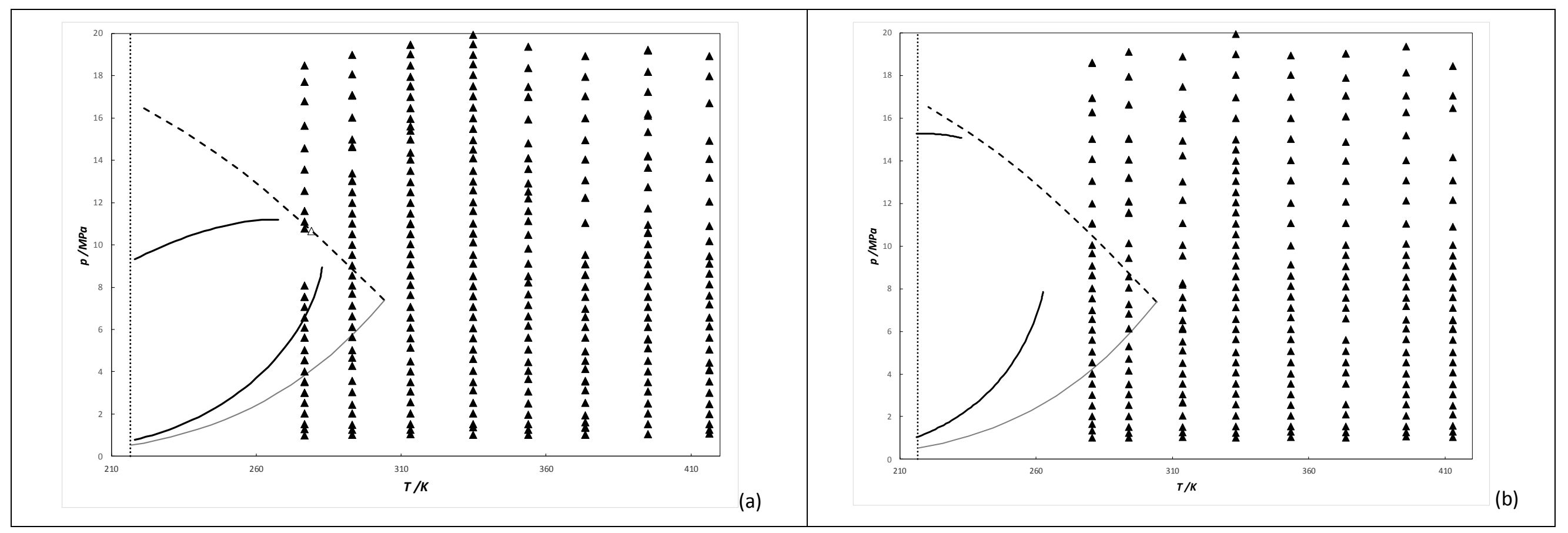




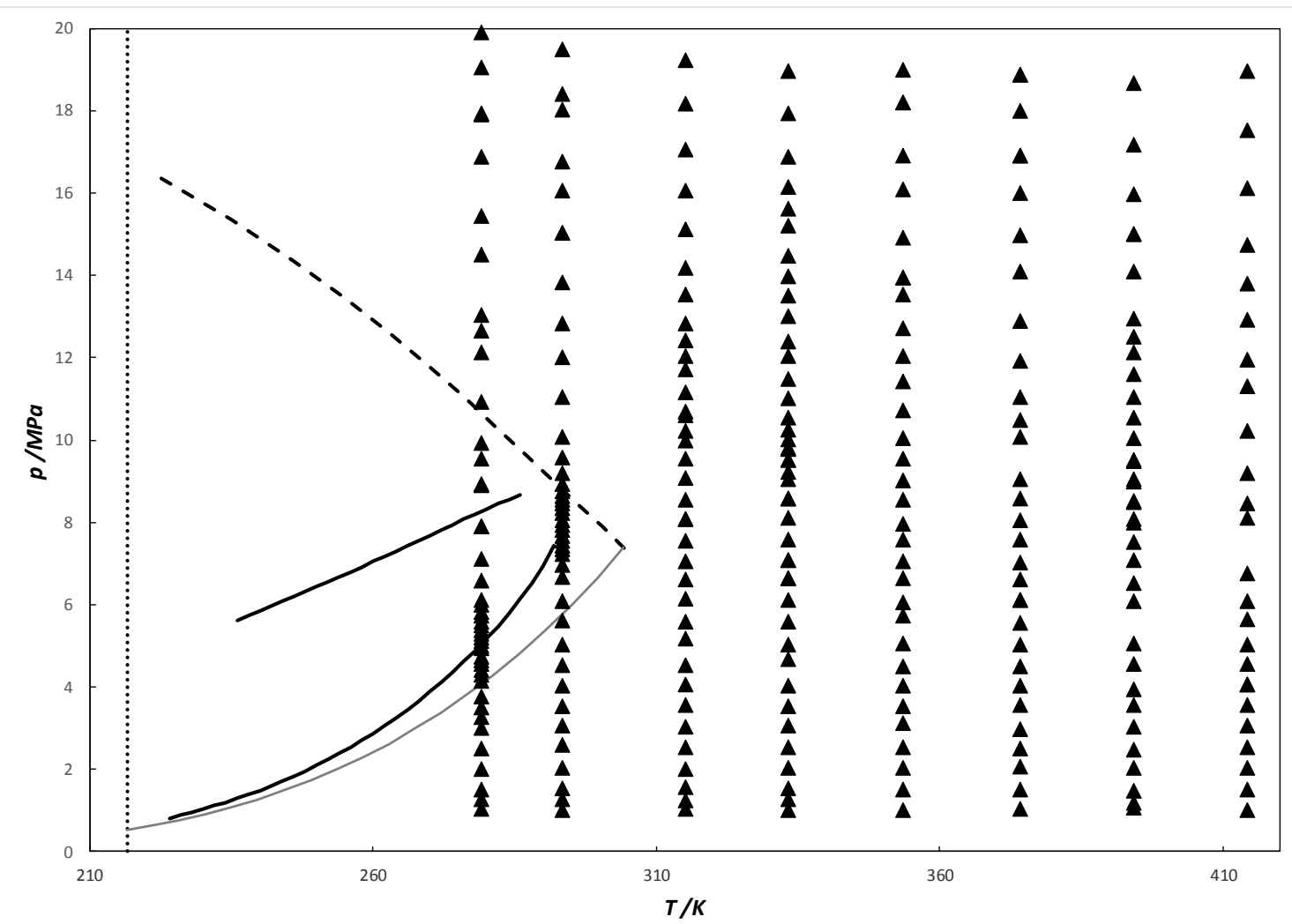

Figure 4: Pressure Temperature envelope of the $\mathrm{CO}_{2}-\mathrm{O}_{2}$ binary system calculated using Peng Robinson EoS (compositions listed in Table 2) showing the experimental points measured ( $\triangle$ ). (a): Mixture 1, (b): mixture 2, (c): Mixture 3. Grey solid line: Pure $\mathrm{CO}_{2}$ vapor pressure. Bold dashed line: mixture critical points line. 


\subsection{Comparison with experimental density data}

The PR EoS previously developed is used to predict the experimental data. Table 9 summarizes the $A A D$ (Eq. 10) and Maximum Absolute Deviation (MAD) in the gas, liquid and supercritical regions. The results (pressure $v s$. molar volume and compressibility factor) for the different mixtures are presented in Figures 5 to 7.

$A A D(\%)=N^{-1} \sum_{i=1}^{N}\left(\left|\rho_{\text {Exp. }}-\rho_{\text {Model }}\right| / \rho_{\text {Exp. }}\right) \times 100$

As it can be seen, the cubic EoS represents the experimental data with satisfactory deviations. As the compressibility factor tends to 1 when the pressure tends to 0 (ideal gas law), it is possible to evaluate the second virial coefficient of the $\mathrm{CO}_{2}-\mathrm{O}_{2}$ binary mixture from the measured compressibility factor. Indeed, $Z=1+\frac{B P}{R T}$ for low to moderate pressure. It is also a good test to evaluate the consistency of the data at low pressure. In addition, we can observe that the maximum of deviation occurs for the low-pressure measurements ( $p<1.5 \mathrm{MPa}$ due to VTD precision) and close to bubble or dew points (possible metastable states). As the compressibility depends on the temperature, the density and the pressure, the uncertainties of the compressibility are therefore expressed using Eq. (11). It can be observed that the compressibility factor "concentrates" all the uncertainties of the measurements. The average value of $u(Z) / Z$ is around $5 \%$.

$u(Z)=Z \sqrt{\left(\frac{u(\rho)}{\rho}\right)^{2}+\left(\frac{u(T)}{T}\right)^{2}+\left(\frac{u(P)}{P}\right)^{2}}$ 


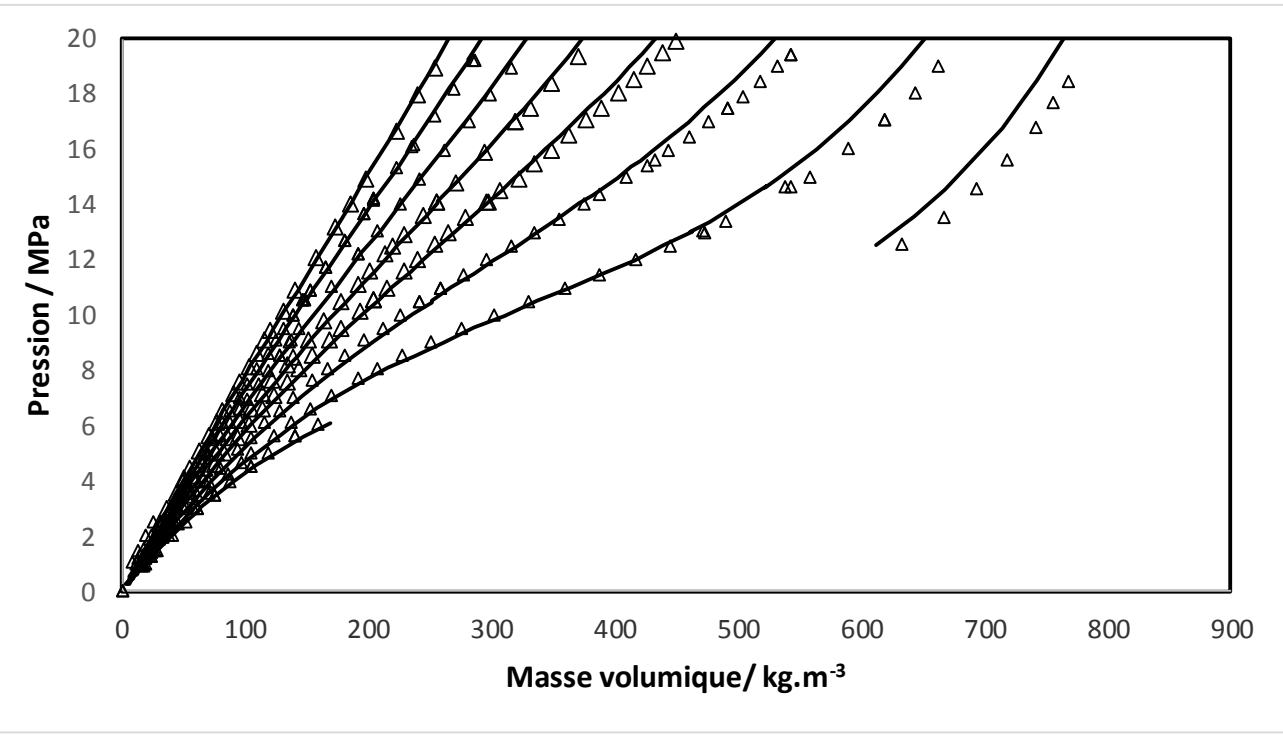

A

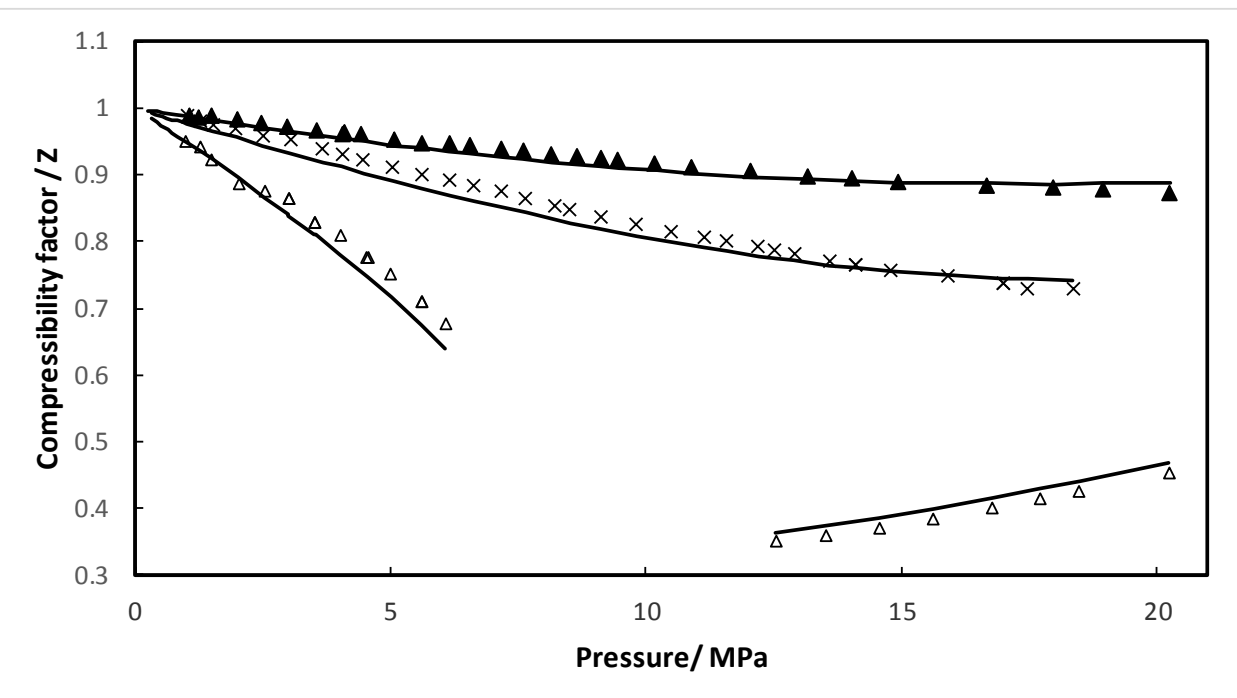

B

Figure 5: A: Pressure as a function of molar density for the $\mathrm{O}_{2}+\mathrm{CO}_{2}$ (mixture 1: 0.274/0.726) binary system. B: Compressibility factor as a function of pressure ( $\Delta$ ): 276.59 K, ( $\times$ ): 353.78 K, ( $\triangle$ ):416.38 K. Solid line: Peng Robinson EoS with Wong Sandler mixing rules and NRTL Activity coefficient model. 


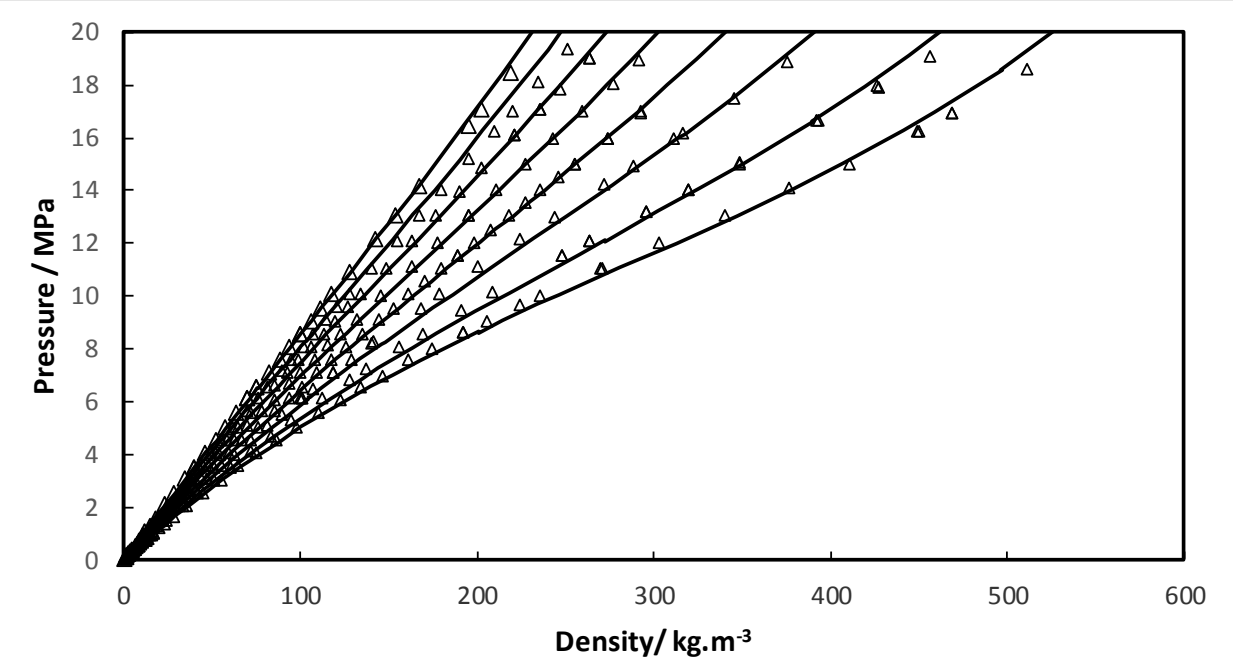

A

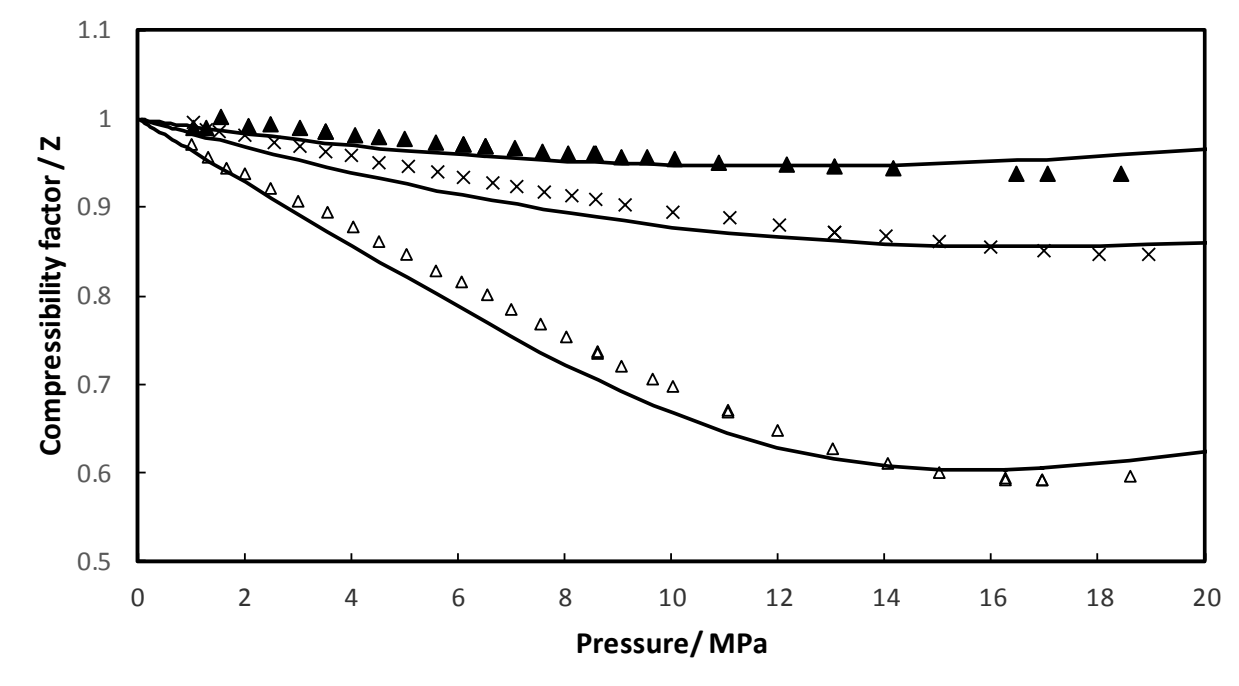

B

Figure 6: A: Pressure as a function of molar density for the $\mathrm{O}_{2}+\mathrm{CO}_{2}$ (mixture 2: $\left.0.483 / 0.517\right)$ binary system. B: Compressibility factor as a function of pressure ( $\Delta$ ): 280.63 K, ( $\times$ ): 353.46 K, ( $\triangle$ ):412.85 K. Solid line: Peng Robinson EoS with Wong Sandler mixing rules and NRTL Activity coefficient model. 


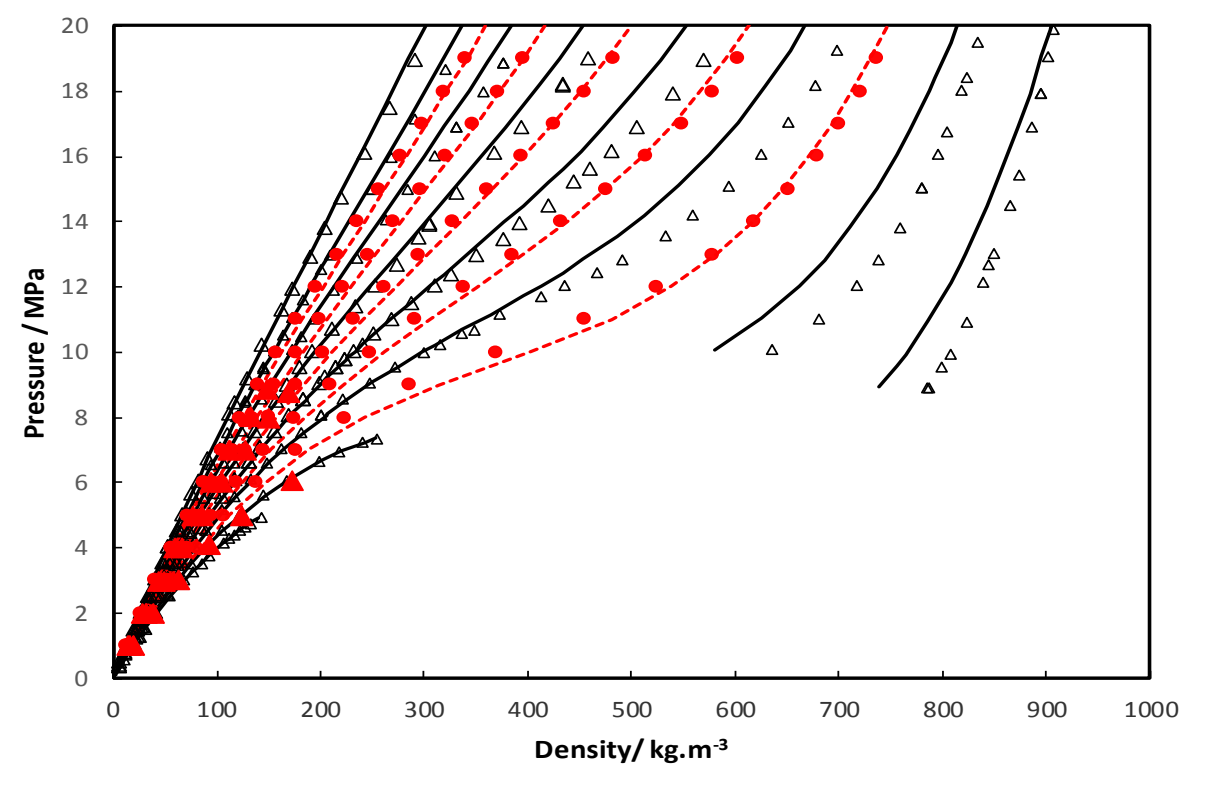

A

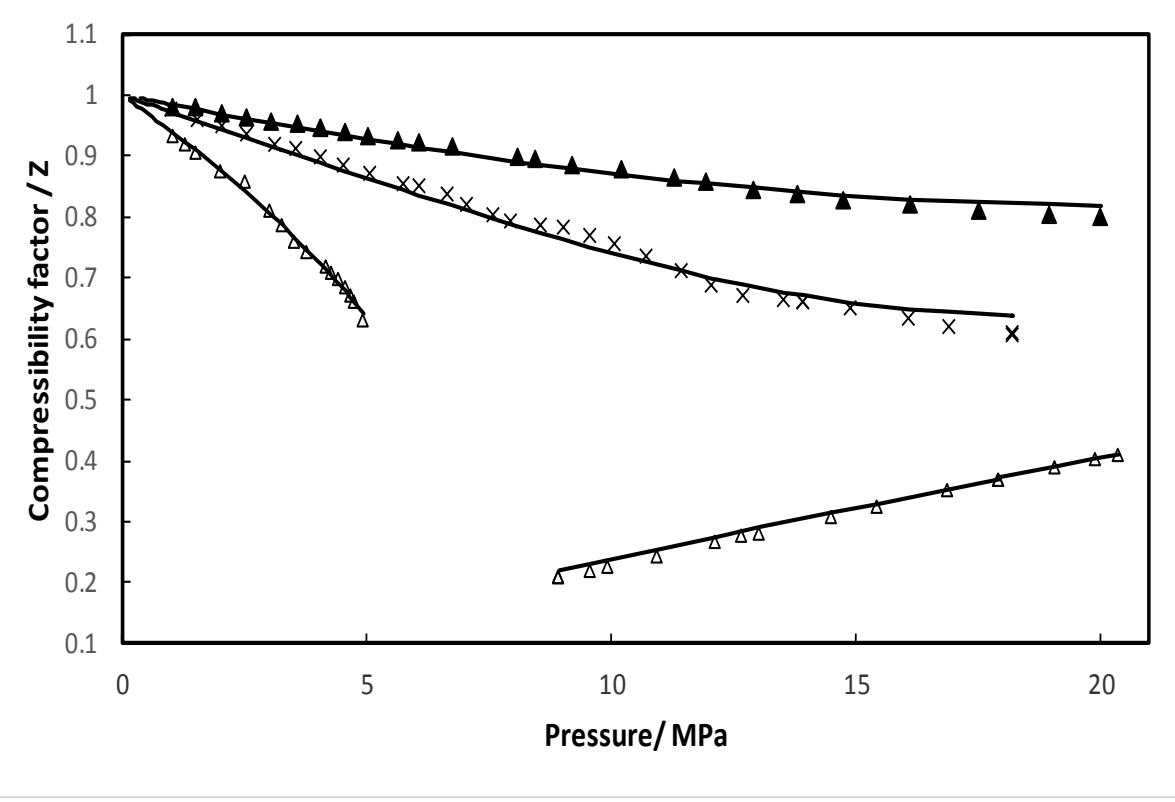

B 


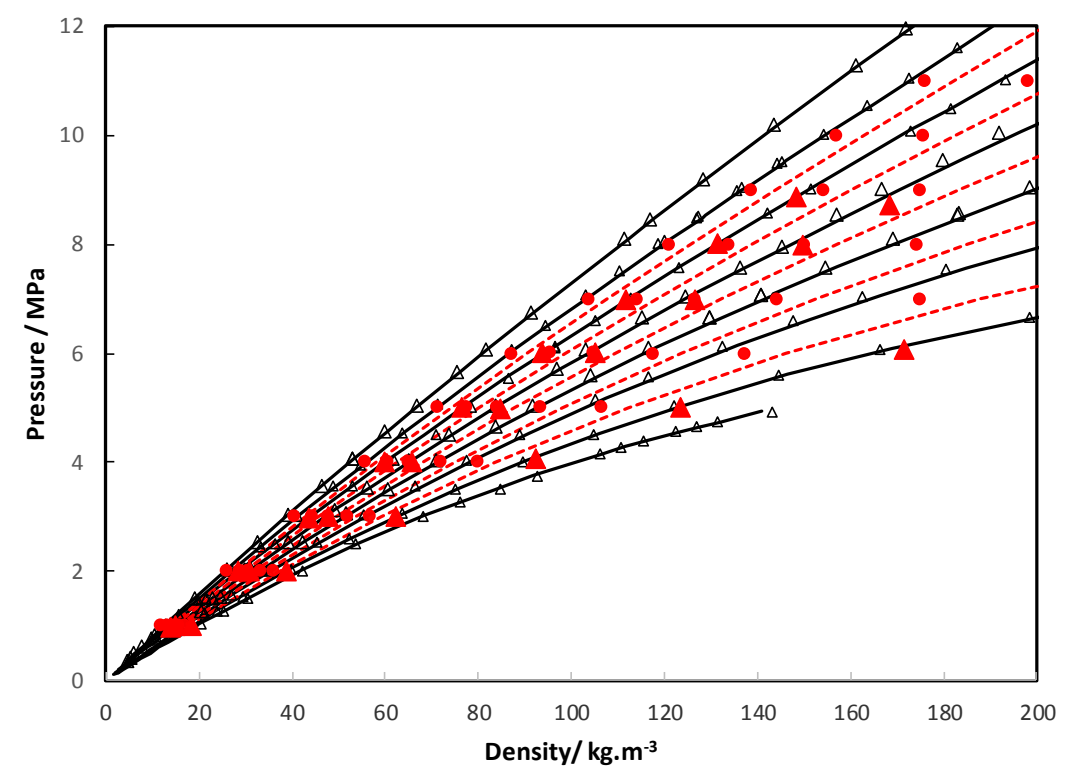

C

Figure 7: A: Pressure as a function of molar density for the $\mathrm{O}_{2}+\mathrm{CO}_{2}$ (mixture 3: 0.128/0.872) binary system (C: zoom in the low pressure region). ( $(\Delta)$ : this work.

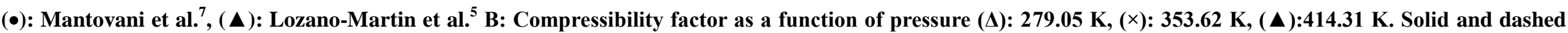
line: Peng Robinson EoS with Wong Sandler mixing rules and NRTL Activity coefficient model. 


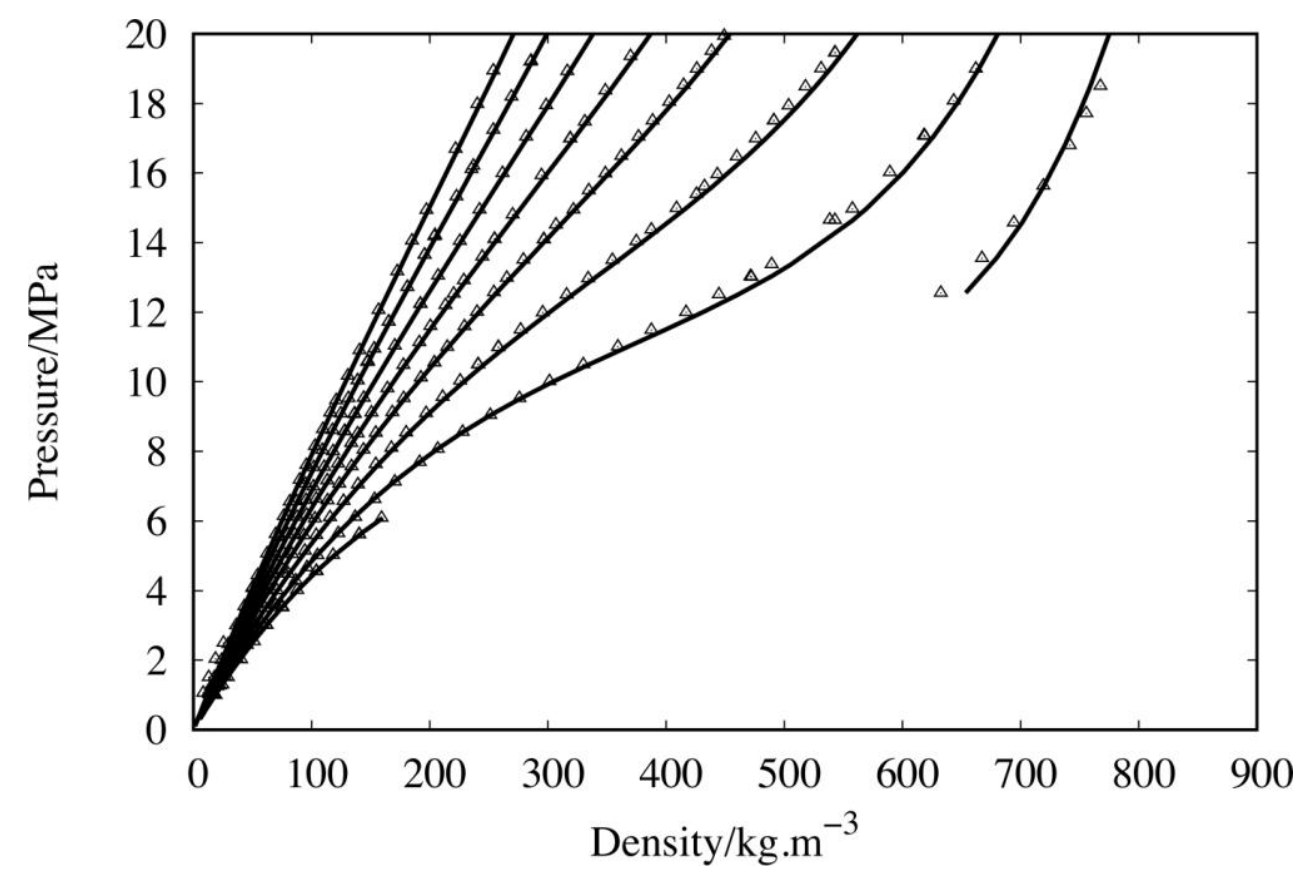

A

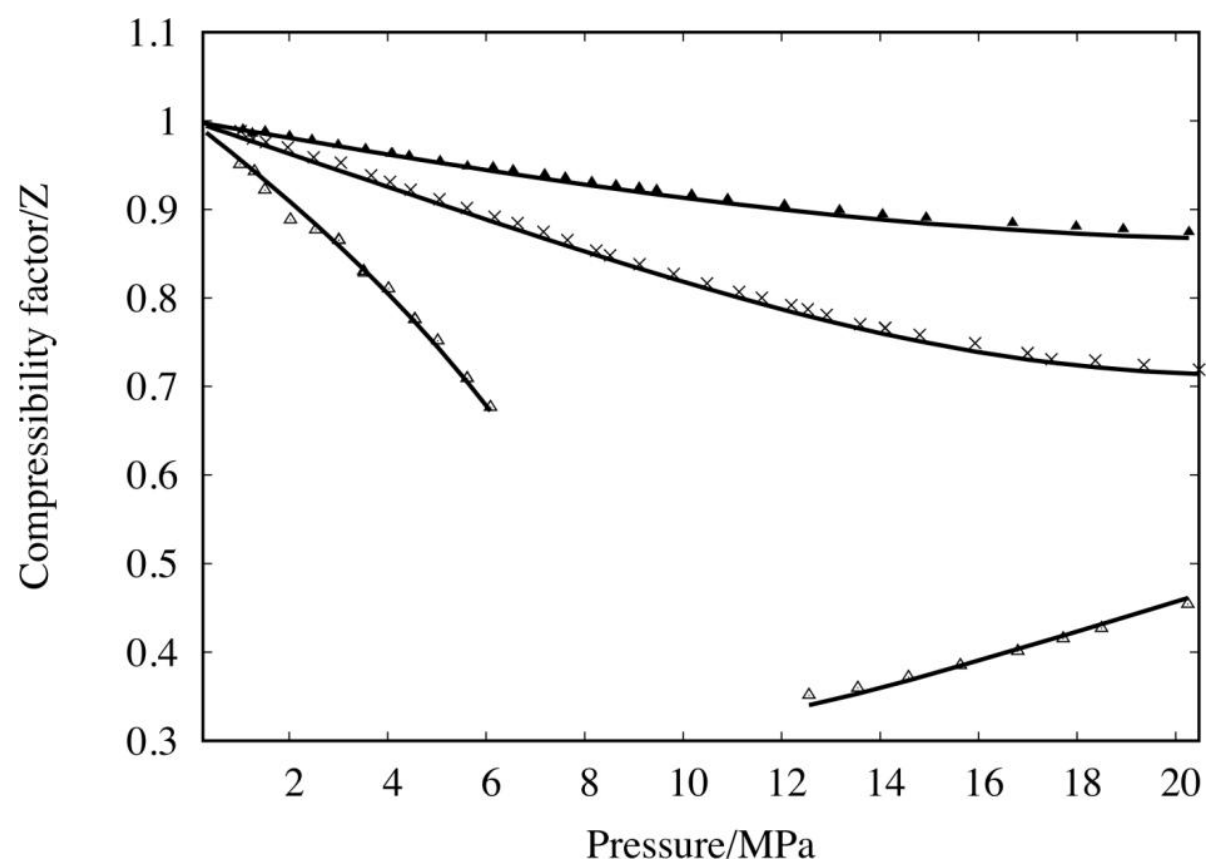

B

Figure 8: A: Pressure as a function of density for the $\mathrm{CO}_{2}+\mathrm{O}_{2}$ (mixture 1: 0.726/0.274) binary system. B: Compressibility factor as a function of pressure ( $\Delta$ ): 276 . $50 \mathrm{~K},(\times)$ : $353.78 \mathrm{~K},(\triangle)$ : $416.38 \mathrm{~K}$. Solid line: EOS-CG. 

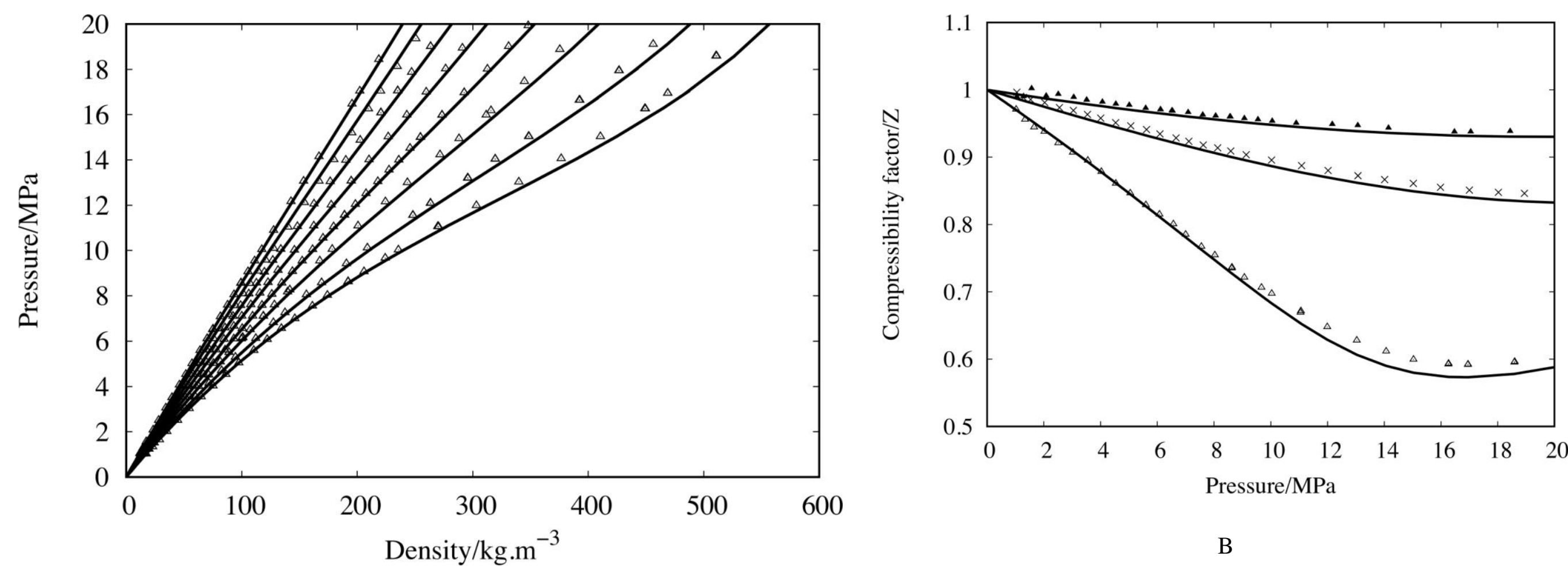

B

A

Figure 9: A: Pressure as a function of density for the $\mathrm{CO}_{2}+\mathrm{O}_{2}$ (mixture 2: 0.517/0.483) binary system. B: Compressibility factor as a function of pressure ( $\Delta$ ): $280.63 \mathrm{~K},(\mathrm{x})$ : $353.46 \mathrm{~K},(\triangle)$ ) $412.85 \mathrm{~K}$. Solid line: EOS-CG. 


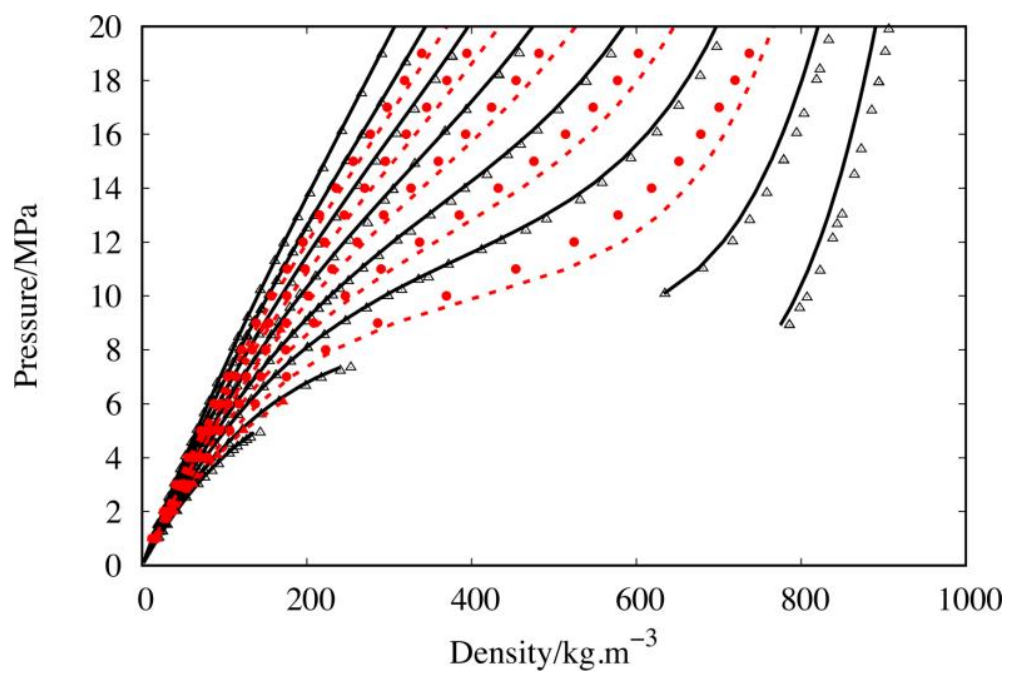

A

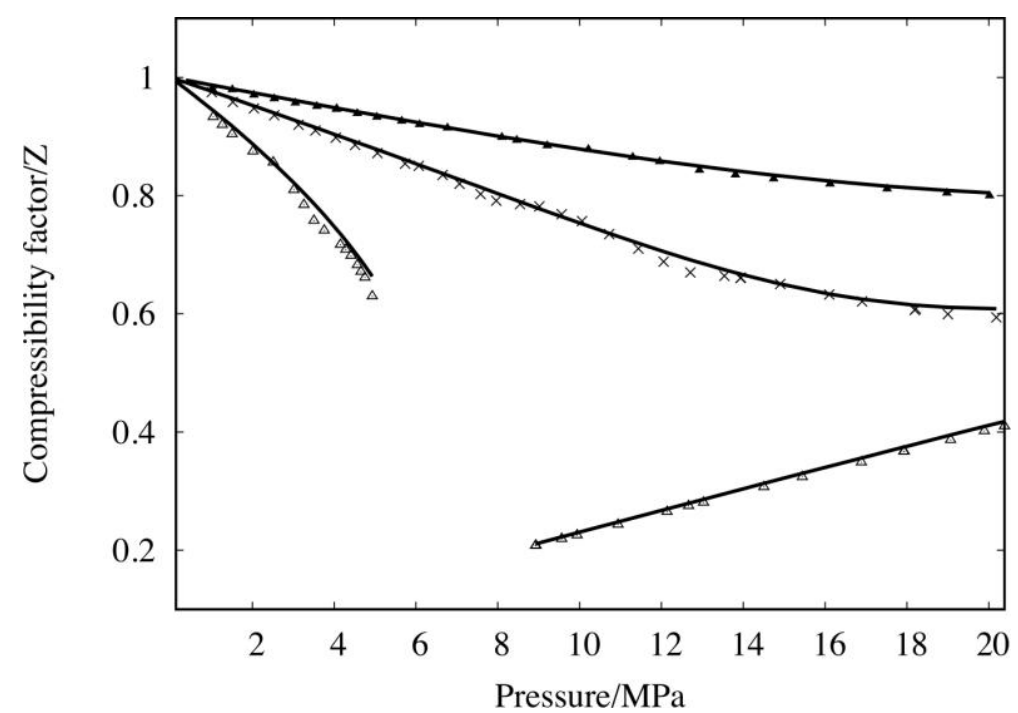

B 


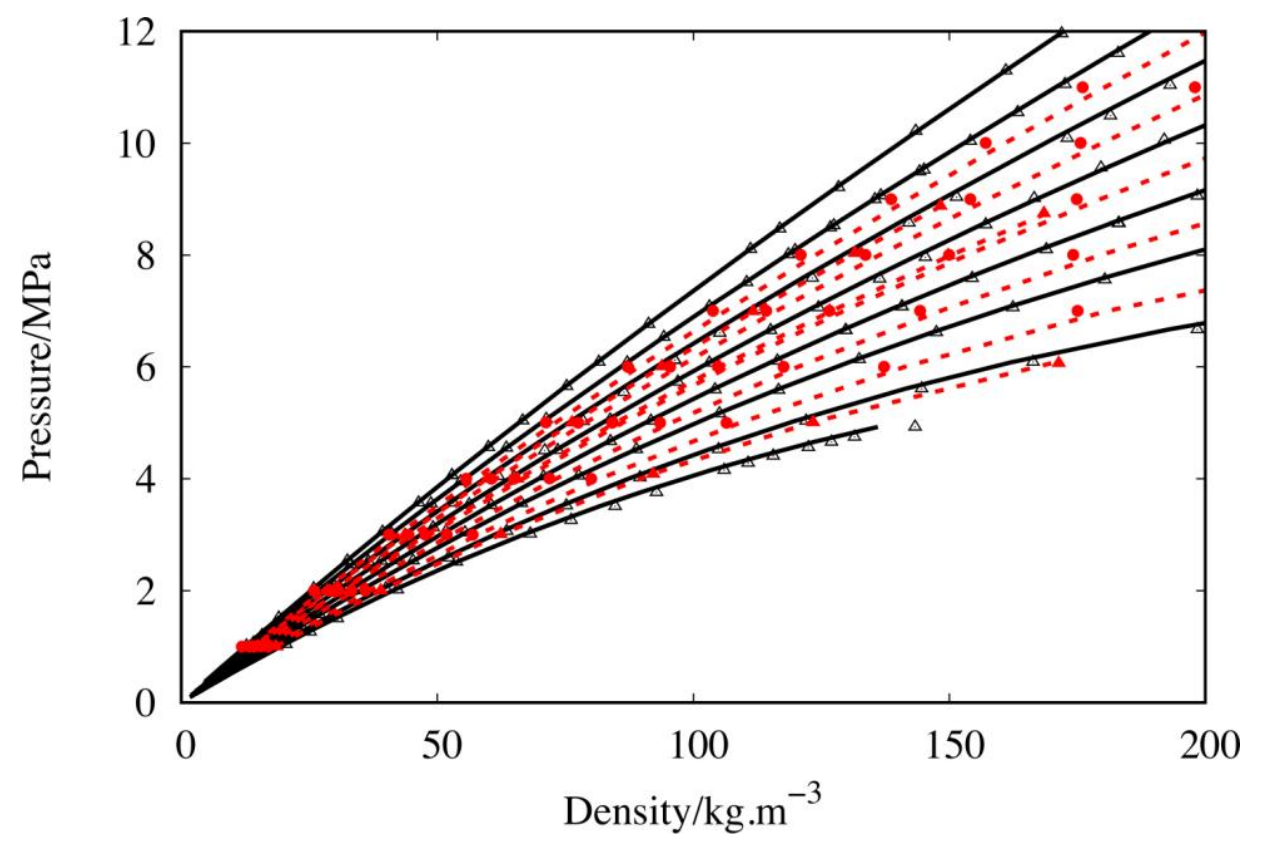

C

Figure 10: A: Pressure as a function of density for the $\mathrm{CO}_{2}+\mathrm{O}_{2}$ (mixture 3: 0.872/0.128) binary system (C: zoom in the low pressure region). ( $\Delta$ ): this work. (๑):

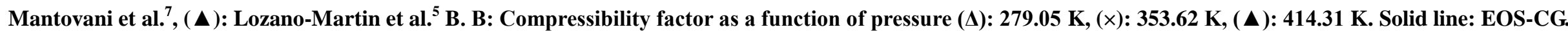


Experimental density data were also compared to a recent EoS, EOS-CG, based on GERG$2008^{4,15}$. Parameters for the reduced mixture density and inverse reduced mixture temperature are provided by Gernert and $\operatorname{Span}^{8}$, but the weighting factor $F_{i j}$ is set to 0 for the binary system $\mathrm{CO}_{2}-\mathrm{O}_{2}$. As a result, the residual mixture behavior only includes the residual behavior of the pure components, weighted by their molar fraction. The reducing functions for density and temperature are presented in Eqs. (11) and (12), where $N$ is the number of components, $T_{C, i}$ is the critical temperature of component $i$ and $\rho_{C, i}$ is the critical density of component $i$.

$\frac{1}{\rho_{r}(x)}=\sum_{i=1}^{N} x_{i}^{2} \frac{1}{\rho_{c, i}}+\sum_{i=1}^{N-1} \sum_{j=i+1}^{N} 2 x_{i} x_{j} \beta_{v, i j} \gamma_{v, i j} \frac{x_{i}+x_{j}}{\beta_{v, i j}^{2} x_{i}+x_{j}} \cdot \frac{1}{8}\left(\frac{1}{\rho_{c, i} i^{\frac{1}{3}}}+\frac{1}{\rho_{c, j}{ }^{\frac{1}{3}}}\right)^{3}$

$T_{r} \overline{(x)}=\sum_{i=1}^{N} x_{i}^{2} T_{c, i}+\sum_{i=1}^{N-1} \sum_{j=i+1}^{N} 2 \beta_{T, i j} \gamma_{T, i j} \cdot\left(T_{c, i} \cdot T_{c, j}\right)^{0.5} x_{i} x_{j} \frac{x_{i}+x_{j}}{\beta_{T, i j}{ }^{2} x_{i}+x_{j}}$

$\beta_{v, i j}, \gamma_{v, i j}, \beta_{T, i j}$ and $\gamma_{T, i j}$ are binary interaction parameters from GERG-2008, 15 and presented in Table 10 for the system $\mathrm{CO}_{2}-\mathrm{O}_{2}$.

As shown in Table 9, EoS-CG predicts the densities more accurately than the PR EoS developed in this work. It should be noted that EOS-CG is based on multi-fluid approximations and that different types of data ( $P \rho T$, VLE, speed of sound, etc.) are used for the development of binary model parameters. Figures 8 - 10 compare experimental and predicted densities and compressibility factors. Due to the use of different data types, rather than only VLE as PR EoS, the phase equilibrium prediction is less accurate for EOS-CG than for PR EoS (see Figure 3). This is also influenced by the presence of a mixture critical point.

Finally, we have compared our experimental results with literature data for compositions similar to ours, i.e. mixture 3. We have plotted the literature data in Figures 7 and 10 (we have also included a zoom to better visualize the low-pressure region). Deviations between these data and PR EoS and EOS-CG are presented in Table 9. As can seen, the order of magnitude of the deviations between our models and experimental data (and our data and literature data) are very close. We can observe a good agreement between the different sets of data. 
Table 9: Deviations between experimental and calculated data using Peng Robinson EoS with the Wong Sandler mixing rules involving NRTL activity coefficient model, and using EoS-CG. Vap: vapor phase, Liq: liquid phase and SC: supercritical region.

\begin{tabular}{|c|c|c|c|c|c|c|c|c|c|c|c|c|}
\hline \multirow[b]{2}{*}{$T / \mathrm{K}$} & \multicolumn{6}{|c|}{ Peng Robinson EoS } & \multicolumn{6}{|c|}{ EoS-CG } \\
\hline & $\begin{array}{c}A A D / \% \\
\text { Vap }\end{array}$ & $\begin{array}{c}A A D / \% \\
\text { Liq }\end{array}$ & $\begin{array}{c}A A D / \% \\
\mathrm{SC}\end{array}$ & $\begin{array}{c}\text { MAD\% } \\
\text { Vap }\end{array}$ & $\begin{array}{c}\text { MAD\% } \\
\text { Liq }\end{array}$ & $\begin{array}{c}M A D \% \\
\text { SC }\end{array}$ & $\begin{array}{c}A A D / \% \\
\text { Vap }\end{array}$ & $\begin{array}{c}A A D / \% \\
\text { Liq }\end{array}$ & $\begin{array}{c}A A D / \% \\
\mathrm{SC}\end{array}$ & $\begin{array}{c}\text { MAD\% } \\
\text { Vap }\end{array}$ & $\begin{array}{c}M A D \% \\
\text { Liq }\end{array}$ & $\begin{array}{c}\text { MAD\% } \\
\text { SC }\end{array}$ \\
\hline \multicolumn{13}{|c|}{ Mixture 1} \\
\hline 293.18 & & & 3.2 & & & 4.5 & & & 1.3 & & & 3.8 \\
\hline 313.18 & & & 2.0 & & & 3.9 & & & 1.5 & & & 2.2 \\
\hline 334.78 & & & 2.2 & & & 3.9 & & & 0.6 & & & 1.3 \\
\hline 395.18 & & & 1.3 & & & 2.1 & & & 0.6 & & & 0.7 \\
\hline 416.38 & & & 0.9 & & & 1.4 & & & 0.4 & & & 0.9 \\
\hline \multicolumn{13}{|c|}{ Mixture 2} \\
\hline 280.63 & & & 2.8 & & & 4.6 & & & 1.6 & & & 3.7 \\
\hline 293.92 & & & 2.7 & & & 4.7 & & & 2.1 & & & 3.4 \\
\hline 313.63 & & & 3.1 & & & 4.4 & & & 2.1 & & & 2.8 \\
\hline 412.85 & & & 1.1 & & & 2.8 & & & 0.7 & & & 1.2 \\
\hline \multicolumn{13}{|c|}{ Mixture 3} \\
\hline 279.05 & 3.0 & 3.7 & & 12.6 & 8.2 & & 2.3 & 1.8 & & 5.1 & 2.0 & \\
\hline 293.31 & & & 3.7 & & & 8.9 & 2.2 & 1.7 & & 5.0 & 2.1 & \\
\hline 315.06 & & & 3.5 & & & 8.6 & & & 1.1 & & & 3.1 \\
\hline 333.25 & & & 2.3 & & & 7.1 & & & 0.9 & & & 2.3 \\
\hline 353.62 & & & 1.6 & & & 6.8 & & & 0.9 & & & 3.1 \\
\hline
\end{tabular}




\begin{tabular}{|c|c|c|c|c|}
\hline 374.23 & 1.4 & 4.5 & 1.7 & 5.1 \\
\hline 394.15 & 1.0 & 3.0 & 0.3 & 0.8 \\
\hline \multicolumn{5}{|c|}{ Lozano-Martín et al. ${ }^{5}, x_{O 2}=0.099856-$ Maximum pressure: $9 \mathrm{MPa}$} \\
\hline 349.92 & 1.78 & 2.5 & 0.2 & 0.3 \\
\hline 374.91 & 1.28 & 1.7 & 0.07 & 0.2 \\
\hline \multicolumn{5}{|c|}{ Mantovani et al. ${ }^{7}, x_{O 2}=0.1291-$ Maximum pressure: $20 \mathrm{MPa}$} \\
\hline 343.15 & 3.34 & 7.55 & 3.4 & 7.1 \\
\hline 363.15 & 3.02 & 8.20 & 3.0 & 7.8 \\
\hline 383.14 & 3.76 & 15.18 & 3.6 & 14.8 \\
\hline
\end{tabular}


Table 10: Values of EoS-CG ${ }^{4}$ binary interaction parameters.

\begin{tabular}{ccccc}
\hline System & $\boldsymbol{\beta}_{\boldsymbol{T}}$ & $\gamma_{\boldsymbol{T}}$ & $\boldsymbol{\beta}_{\boldsymbol{v}}$ & $\boldsymbol{\gamma}_{\boldsymbol{v}}$ \\
\hline $\mathrm{CO}_{2}+\mathrm{O}_{2}$ & 1.0 & 1.032 & 1.0 & 1.0845 \\
\hline
\end{tabular}

\section{Conclusion}

The densities of three $\mathrm{CO}_{2}-\mathrm{O}_{2}$ binary mixtures were measured using VTD densitometer, Anton Paar DMA 512, in the gas, liquid and supercritical regions. The densitometer was first calibrated using pure $\mathrm{CO}_{2}$ and the FPMC calibration technique. The maximum expanded uncertainties on temperature, pressure and densities are $U(p)=0.0005 \mathrm{MPa}, U(T)=0.3 \mathrm{~K}$ and $U(\rho)=15 \mathrm{~kg} \cdot \mathrm{m}^{-3}$, respectively. The highest uncertainties were observed at very low pressure conditions in the gas phase or in the vicinity of the bubble point curve in the liquid phase. The measured densities were employed to evaluate the classical Peng-Robinson cubic EoS with parameters adjusted on VLE data from the literature. The model gives satisfactory results in the prediction of the volumetric properties in the typical conditions of storage in salt caverns. However, the GERG-2008-based EoS-CG was more accurate with AAD of only $1.1 \%$ and MAD of $5.1 \%$. The main advantage of EOS based on a thermodynamic potential is that all thermodynamic properties of a mixture or pure substance can be consistently derived from the potential; these properties are needed in the assessment of cavern stability.

\section{Acknowledgments}

Financial support from Agence Nationale de la Recherche (ANR) through the project FluidSTORY ( $\mathrm{n}^{\circ} 7747$, ID ANR-15-CE06-0015) is gratefully acknowledged.

\section{References}

1. Götz, M.; Lefebvre, J.; Mörs, F.; Koch, A. M.; Graf, F.; Bajohr, S.; Reimert, R.; Kolb, T., Renewable Power-to-Gas: A technological and economic review. Renewable energy 2016, 85, 1371-1390. 
2. Kezibri, N.; Bouallou, C., Conceptual design and modelling of an industrial scale power to gas-oxy-combustion power plant. Int. J. Hydrogen Energy 2017, 42, (30), 1941119419.

3. Blanco-Martín, L.; Rouabhi, A.; Hadj-Hassen, F., Use of salt cavern in the energy transition context: application to the Power-to-Gas- Oxyfuel process. SN Appl. Sci. 2020, submitted.

4. Li, H.; Dong, B.; Yu, Z.; Yan, J.; Zhu, K., Thermo-physical properties of $\mathrm{CO}_{2}$ mixtures and their impacts on $\mathrm{CO} 2$ capture, transport and storage: progress since 2011. Appl. Energy 2019, 255, 113789.

5. Lozano-Martin, D.; Akubue, G.U.; Moreau, A.; Tuma, D.; Chamorro, C.R., Accurate experipmental $(\mathrm{p}, \rho, \mathrm{T})$ data of the $(\mathrm{CO} 2+\mathrm{O} 2)$ binary system for the development of models for CCs processes. J. Chem. Thermodyn. 2020, 150, 106210.

6. Commodore, J.A.; Deering, C.E.; Marriott, R.A., Volumetric properties and phase behavior of sulfur dioxide, carbon disulfide and oxygen in high-pressure carbon dioxide fluid. Fluid Phase Equilib. 2018, 477, 30-39.

7. Mantovani, M.; Chiesa, P.; Valenti, G.; Gatti, M.; Consonni, S., Supercritical pressuredensity-temperature measurements on $\mathrm{CO} 2-\mathrm{N} 2, \mathrm{CO} 2-\mathrm{O} 2$ and $\mathrm{CO} 2-\mathrm{Ar}$ binary mixtures. $J$. Supercrit. Fluids 2012, 61-34-43.

8. Gernert, J.; Span, R., EOS-CG: A Helmholtz energy mixture model for humid gases and CCS mixtures. J. Chem. Thermodyn. 2016, 93, 274-293.

9. Rivollet, F.; Jarne, C.; Richon, D., P $\rho T$ and VLE for Ethane+ Hydrogen Sulfide from (254.05 to 363.21) K at Pressures up to 20 MPa. J Chem. Eng. Data 2005, 50, (6), 1883-1890.

10. Coquelet, C.; Ramjugernath, D.; Madani, H.; Valtz, A.; Naidoo, P.; Meniai, A. H., Experimental measurement of vapor pressures and densities of pure hexafluoropropylene. $J$ Chem. Eng. Data 2010, 55, (6), 2093-2099.

11. Nazeri, M.; Chapoy, A.; Valtz, A.; Coquelet, C.; Tohidi, B., Densities and derived thermophysical properties of the $0.9505 \mathrm{CO} 2+0.0495 \mathrm{H} 2 \mathrm{~S}$ mixture from $273 \mathrm{~K}$ to $353 \mathrm{~K}$ and pressures up to $41 \mathrm{MPa}$. Fluid Phase Equilib. 2016, 423, 156-171.

12. Bouchot, C.; Richon, D., An enhanced method to calibrate vibrating tube densimeters. Fluid phase Equilib. 2001, 191, (1-2), 189-208.

13. Khalil, W.; Coquelet, C.; Richon, D., High-pressure Vapor- Liquid equilibria, liquid densities, and excess molar volumes for the carbon dioxide+ 2-propanol system from (308.10 to 348.00) K. J. Chem. Eng. Data 2007, 52, (5), 2032-2040.

14. Lemmon, E.; Bell, I. H.; Huber, M.; McLinden, M., NIST Standard Reference Database 23: Reference Fluid Thermodynamic and Transport Properties-REFPROP, Version 10.0, National Institute of Standards and Technology. 2018. URL http://www.nist. gov/srd/nist23. cfm.

15. Span, R.; Wagner, W., A new equation of state for carbon dioxide covering the fluid region from the triple-point temperature to $1100 \mathrm{~K}$ at pressures up to $800 \mathrm{MPa}$. J. Phys. Chem. Ref. Data 1996, 25, (6), 1509-1596.

16. Peng, D.-Y.; Robinson, D. B., A new two-constant equation of state. IEC Fund. 1976, $15,(1), 59-64$. 
17. Wong, D. S. H.; Sandler, S. I., A theoretically correct mixing rule for cubic equations of state. AIChE J. 1992, 38, (5), 671-680.

18. Renon, H.; Prausnitz, J. M., Local compositions in thermodynamic excess functions for liquid mixtures. AIChE J. 1968, 14, (1), 135-144.

19. Fredenslund, A.; Sather, G., Gas-liquid equilibrium of the oxygen-carbon dioxide system. J. Chem. Eng. Data 1970, 15, (1), 17-22.

20. Kaminishi, G.-i.; Toriumi, T., Gas-liquid equilibrium under high pressures. VI. Vaporliquid phase equilibrium in the $\mathrm{CO} 2-\mathrm{H} 2, \mathrm{CO} 2-\mathrm{N} 2$, and $\mathrm{CO} 2-\mathrm{O} 2$ systems. Kogyo Kagaku Zasshi 1966, 69, (2), 175-178.

21. Muirbrook, N.; Prausnitz, J., Multicomponent vapor-liquid equilibria at high pressures: Part I. Experimental study of the nitrogen—oxygen—carbon dioxide system at $0^{\circ}$ C. AIChE J. 1965, 11, (6), 1092-1096.

22. Zenner, G.H.; Dana, L.I Liquid-vapor equilibrium compositions of carbon dioxideoxygen-nitrogen mixtures, Chemical engineering progress symposium series, 1963, 36-41.

23. Lasala, S.; Chiesa, P.; Privat, R.; Jaubert, J.-N., VLE properties of CO2-Based binary systems containing N2, O2 and Ar: Experimental measurements and modelling results with advanced cubic equations of state. Fluid Phase Equilib. 2016, 428, 18-31.

24. Westman, S. F.; Stang, H. J.; Løvseth, S. W.; Austegard, A.; Snustad, I.; Ertesvåg, I. S., Vapor-liquid equilibrium data for the carbon dioxide and oxygen $(\mathrm{CO} 2+\mathrm{O} 2)$ system at the temperatures 218, 233, 253, 273, 288 and $298 \mathrm{~K}$ and pressures up to $14 \mathrm{MPa}$. Fluid Phase Equilib. 2016, 421, 67-87. 


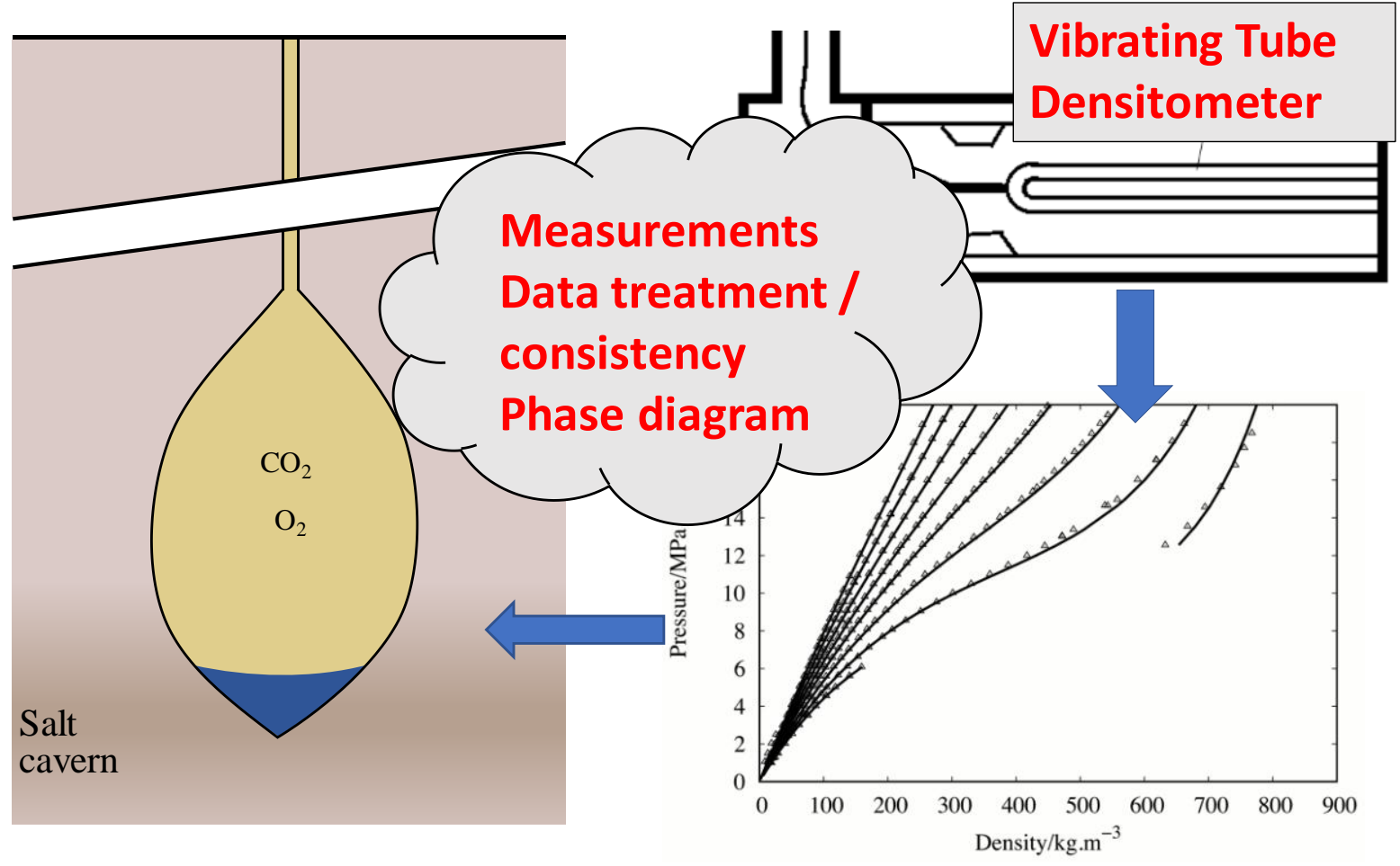

